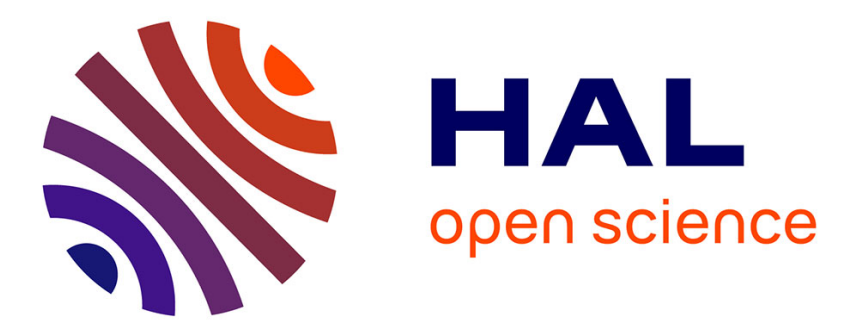

\title{
Algebraic Algorithms to Separate Overlapping Secondary Surveillance Radar Replies
}

Nicolas Petrochilos, Alle-Jan van Der Veen

\section{To cite this version:}

Nicolas Petrochilos, Alle-Jan van Der Veen. Algebraic Algorithms to Separate Overlapping Secondary Surveillance Radar Replies. IEEE Transactions on Signal Processing, 2007, 55 (7), pp.3746 - 3759. 10.1109/TSP.2007.894248. hal-01693636

\section{HAL Id: hal-01693636 \\ https://hal.univ-reims.fr/hal-01693636}

Submitted on 7 Dec 2019

HAL is a multi-disciplinary open access archive for the deposit and dissemination of scientific research documents, whether they are published or not. The documents may come from teaching and research institutions in France or abroad, or from public or private research centers.
L'archive ouverte pluridisciplinaire HAL, est destinée au dépôt et à la diffusion de documents scientifiques de niveau recherche, publiés ou non, émanant des établissements d'enseignement et de recherche français ou étrangers, des laboratoires publics ou privés. 


\title{
Algebraic algorithms to separate overlapping Secondary Surveillance Radar replies
}

\author{
Nicolas Petrochilos ${ }^{* 1}$ and Alle-Jan van der Veen ${ }^{2}$ \\ (1) Dept. Electrical Engineering, University of Hawai'i at Mānoa \\ 2540 Dole Street, Honolulu, HI 96822 \\ (2) Dept. Electrical Engineering, Delft University of Technology, \\ Mekelweg 4, 2628 CD Delft, The Netherlands \\ petro@ieee.org, a.j.vanderveendtudelft.nl
}

EDICS: SAM-APPL, SSP-SSEP

\begin{abstract}
The Secondary Surveillance Radar (SSR) is a transponder system used in air traffic control. Due to growing traffic densities, it is increasingly likely that a ground station receives a mixture of responses of various aircraft, partly overlapping in frequency and time. Currently such "collisions" are disregarded, at a loss of system performance and reliability. In this article, we propose to equip the ground station with an antenna array, and investigate techniques to blindly separate such a mixture based on source waveform properties. At base-band, a received SSR signal consists of a binary sequence with alphabet $\{0,1\}$, modulated by a complex exponential due to the residual carrier frequency. We present three algebraic algorithms to compute the separating beamformers by taking into account the particular modulation format of the received signal. The CramerRao Bound is derived, extensive simulations are presented, and an experimental platform has been built to collect measurement data and demonstrate the algorithms.
\end{abstract}

\section{INTRODUCTION}

Secondary Surveillance Radar (SSR) is essential for Air Traffic Control (ATC). Unlike the primary radar, the SSR is a communication radar (transponder system) that informs the ATC about the identity and the altitude of aircraft [1]. An SSR ground station uses a rotating scanning beam and transmits interrogating queries, consisting of pulse trains modulated on a carrier at $1030 \mathrm{MHz}$. Upon receiving a query, an aircraft responds by transmitting an SSR reply signal, a bursty pulse train modulating a carrier at $1090 \mathrm{MHz}$ and containing the requested information. The system was designed in the 1950s,

Manuscript received March 29, 2006; revised September 4, 2006.

Alle-Jan van der Veen is with Delft University of Technology, Delft, The Netherlands (e-mail: a.j.vanderveen@tudelft.nl).

Nicolas Petrochilos is with the University of Hawai' i, Hi, the research was performed while he was affiliated with Delft University of Technology, and continued in University of Tor vergata, Roma, Italy (e-mail: petro@ieee.org). but is currently limited by the fact that all replies nominally use the same carrier frequency, and may overlap in time. This occurs, e.g., if two aircraft are close to each other but at different heights, or when an aircraft responds to a query from a neighboring ground station. If two responses overlap, the receiver cannot decode the message and both are lost [2].

To bring some relief, a new protocol (Mode S, for Selective), operating at the same frequencies, is currently being installed [3]. In Mode S, aircraft can be individually addressed to give a response, enabling short data communications between the station and the aircraft. This new mode will also assist the Traffic Advisory and Collision Avoidance System (TCAS) by providing automated communication between the aircraft. Nonetheless, also in this protocol overlaps may occur.

Today, the ground station uses the same rotating antenna for transmission and reception of SSR signals. We may envision two extensions: (1) Create a distributed system, where the existing radar system is used for transmission of the queries only, but where a network of receivers is placed at various locations, (2) Equip each receiver with an array of antennas [4], [5]. This enables multi-lateral location estimation and facilitates message detection [4], [6], [7]. Indeed, at each receiver base station, the overlapping reply signals can be separated using blind beamforming, and subsequently for each recovered signal we can detect the individual symbols and estimate the direction of arrival (DOA) and the Time of Arrival (TOA). The combined information from several receivers allows multi-lateral location estimation at the system level. Estimating the beamformers and the parameters at the receiver is the aim of this paper.

Blind source separation can be done based on properties of the array response matrix or on properties of the source signals. The former has as disadvantage that a carefully cali- 
brated array must be used, and that no multipath is tolerated. Therefore, we consider the rich structure of the source signals as a feature for separation. Indeed, SSR replies have a very structured source model: each sample at the receiver is the product of a binary PAM symbol taken from the alphabet $\{0,1\}$, multiplied by a complex exponential (phase progression) due to the residual carrier frequency.

Blind source separation of SSR reply signals was first considered by Comon in [8]. This algorithm considered maximizing a contrast function based on higher-order statistics (HOS). However, it was noted by Petrochilos and Comon in [9], [10] that such HOS methods are unreliable because for SSR Mode S signals all cumulants of order 3, 4 and 5 have a large probability to be zero. Thus, algorithms using cumulants of 6-th order or higher need to be used.

In this article, we present a collection of algorithms which implicitly use 6-th and 8-th order statistics. The proposed algorithms are algebraic: similar to the Analytic Constant Modulus Algorithm (ACMA) [11], the beamformers are computed from a batch of data by solving a joint diagonalization problem. Some of these algorithms were first presented at conferences [12], [13]. Here, we present the algorithms in a broader perspective, and compare them in simulations and using experimental data. Full details can be found in the $\mathrm{PhD}$ thesis [5]. Follow-up work includes [14], where an alternative time-domain approach has been proposed for the restrictive case of two partly overlapping Mode $\mathrm{S}$ replies with a sufficiently large time difference of arrival. The present paper concentrates on the complementary case where signals are highly overlapping.

The paper is structured as follows. Section II introduces the data model and lists the assumptions and some preliminary material. Section III presents three properties of the source signals, and Section IV uses these to derive three algorithms to find separating beamformers for each of the sources. Section V derives the relevant Cramer-Rao Bound (CRB), and section VI compares the algorithms in simulations. In the course of this work, we have constructed an experimental platform consisting of an array of four antennas. We apply the algorithms to measurement sets collected with the array, and show the results in section VII.

Notation: I denotes the identity matrix, and $\mathbf{0}$ and $\mathbf{1}$ are the vectors with all entries are equal to 0 and 1 , respectively. We denote by $(\cdot)^{*}$ the complex conjugation, by $(\cdot)^{T}$ the matrix transpose, and by $(\cdot)^{H}$ the matrix conjugate transpose.

$\mathrm{E}\{\cdot\}$ denotes the mathematical expectation operator, and $\mathrm{Vec}$ is the operator that stacks the columns of a matrix $\mathbf{A}$ into a single vector a. The notation $(\cdot)^{\dagger}$ refers to the MoorePenrose inverse (pseudo-inverse). The symbol $\odot$ denotes the Schur-Hadamard (element-wise) matrix product, and $\otimes$ the Kronecker product.

\section{DATA MOdEL AND PREliminaries}

SSR communicates via two different protocols: Mode A/C and Mode S. The A/C mode was initiated during World War II, and is supposed to be soon totally replaced by Mode S. In this paper we make the assumption that only Mode $S$ replies are present. A combined model containing both Mode A/C and Mode $\mathrm{S}$ replies is more complex, due to the slightly different (incommensurate) pulse lengths.

\section{A. Received data model}

A Mode $\mathrm{S}$ reply frame contains either 56 or 112 binary symbols $b_{n}$. The bits are encoded in a "Manchester Encoding" scheme, where $b_{n}=0$ is coded as $\mathbf{b}_{n}=$ $[0,1]$, and $b_{n}=1$ as $\mathbf{b}_{n}=[1,0]$. The transmitted bit stream $\mathbf{b}$ is a burst (packet) consisting of a preamble $\mathbf{p}=[1,0,1,0,0,0,0,1,0,1,0,0,0,0,0,0]$ followed by the encoded data bits, i.e.,

$$
\mathbf{b}=\left[\mathbf{p}, \mathbf{b}_{1}, \mathbf{b}_{2}, \cdots, \mathbf{b}_{56 / 112}\right]
$$

with a total length $N \in\{128,240\}$. The preamble is aimed at facilitating the detection of the start of a packet.

The Mode $\mathrm{S}$ reply signal emitted by the aircraft transponder is a pulse amplitude modulation of $\mathbf{b}$, and has the form

$$
b(t)=\sum_{n=0}^{127 / 239} \mathbf{b}[n] p\left(t-n T_{s}\right)
$$

where $\mathbf{b}[n]$ is the $n$-th entry of $\mathbf{b}$, and $p(t)$ is a (nominally) rectangular pulse of width $T_{s}=0.5 \mu \mathrm{s}$, for Mode $\mathrm{S}$.

Before being emitted by the antenna, the signal is upconverted to have a center frequency $f_{e}$. Nominally, this carrier frequency is $f_{c}=1090 \mathrm{MHz}$, but the tolerance permitted by the ICAO is $\pm 3 \mathrm{MHz}$, thus $f_{e} \neq f_{c}$. (In future, this tolerance will be reduced to $\pm 1 \mathrm{MHz}$.) Due to this possible carrier frequency mismatch, a residual frequency $f=f_{e}-f_{c}$ will remain after down-conversion by $f_{c}$ to baseband. This residual carrier adds a significant phase rotation $\exp (j 2 \pi f t)$ to the transmitted symbols.

At the receiver, each antenna signal is downconverted and sampled at rate $1 / T_{s}$. Not assuming temporal synchronization within a symbol period, the received baseband signal $s[n]=$ $s\left(n T_{s}\right)$ is described (up to a complex gain factor) as

$$
s[n]=b[n] \exp \left(\jmath 2 \pi n f T_{s}\right)=b[n] \phi^{n}
$$

where $\phi=\exp \left(\jmath 2 \pi f T_{s}\right)$ is the phase shift due to the residual carrier frequency over a sampling period. This signal is actually multiplied by an unknown complex gain factor representing the effect of the channel, the receiver amplifier, and an initial phase offset. This will be taken into account in the next section.

\section{B. Problem statement}

We extend the single source model (3) to the reception of a mixture of $d$ independent SSR source signals impinging on an $m$-element antenna array. The baseband antenna signals are sampled at rate $1 / T_{s}$ and stacked in vectors $\mathbf{x}[n]$ (size $m$, where the $i$-th entry corresponds to the $i$-th antenna signal). After collecting $N$ samples, the observation model is

$$
\mathbf{X}=\mathbf{M S}+\mathbf{E}
$$

where $\mathbf{X}=[\mathbf{x}[1], \cdots, \mathbf{x}[N]]$ is the $m \times N$ received signal matrix, $\mathbf{M}$ is an unknown $m \times d$ mixing matrix which includes 
the antenna responses, path coefficients, initial phase offsets, the array signatures and the complex gains of the sources, $\mathbf{S}=[\mathbf{s}[1], \cdots, \mathbf{s}[N]]$ is the $d \times N$ source matrix, where $\mathbf{s}[n]=$ $\left[s_{1}[n], \cdots, s_{d}[n]\right]^{T}$ is a stacking of the $d$ source signals, and $\mathbf{E}$ is the $m \times N$ noise matrix. We assume that the sources have unit amplitude and that the noise is temporally and spatially white.

Note that each source $i$ transmits data in packets of finite length $\ell_{i}\left(\ell_{i}=128\right.$ or 240 bits) and with an arbitrary starting time. Each source can thus have an arbitrary time offset $\tau_{i}$ (positive or negative integer) with respect to the start of the observation interval. To consider this effect, we apply zeropadding and truncation operations to the data time series $\mathbf{b}[n]$ in (1) to obtain a similar sequence $\tilde{\mathbf{b}}[n], 1 \leq n \leq N$, where

$$
\tilde{\mathbf{b}}[n]= \begin{cases}0, & n-\tau<1 \text { or } n-\tau>\ell_{i} \\ \mathbf{b}[n-\tau], & 1 \leq n-\tau \leq \ell_{i} .\end{cases}
$$

The source matrix $\mathbf{S}$ has structure

$$
\mathbf{S}=\mathbf{F} \odot \mathbf{B}
$$

where $\odot$ is the Schur-Hadamard (pointwise multiplication) operator, and

$\mathbf{F} \stackrel{\text { def }}{=}\left[\begin{array}{cccc}\phi_{1} & \phi_{1}^{2} & \ldots & \phi_{1}^{T} \\ \vdots & \vdots & & \vdots \\ \phi_{d} & \phi_{d}^{2} & \ldots & \phi_{d}^{T}\end{array}\right]$,

$$
\mathbf{B} \stackrel{\text { def }}{=}\left[\begin{array}{c}
\tilde{\mathbf{b}}_{1}[1] \\
\vdots \\
\tilde{\mathbf{b}}_{d}[1]
\end{array}\right.
$$

Although the algorithms which we will propose will not be based on a calibrated array (i.e., a parametric structure for $\mathbf{M}$ ), it is sometimes interesting to make such an assumption: (1) it allows comparison to source separation algorithms which are based on direction finding, such as MUSIC and ESPRIT, (2) in some cases, the DOAs are also of interest, e.g., for localizing the aircraft (although this is the task of the primary radar), or for assigning each retrieved message to the corresponding aircraft (since the blind source separation algorithms retrieve the messages in an arbitrary ordering). Thus, if the array is a calibrated Uniform Linear Array (ULA) with half-wavelength antenna spacing, and if the multipath is negligible, we can further write $\mathbf{M}=\mathbf{A} \mathbf{G}$ where $\mathbf{A}=\left[\mathbf{a}\left(\theta_{1}\right), \cdots, \mathbf{a}\left(\theta_{d}\right)\right]$ is the $m \times d$ steering matrix, $\theta_{i}$ is the direction of incidence of the $i$-th source with respect to the ULA boresight, $\mathbf{a}(\theta)$, is the array steering vector, defined as

$$
\mathbf{a}(\theta)=[1, \exp (\jmath \pi \cos (\theta)), \cdots, \exp (\jmath \pi(m-1) \cos (\theta))]^{T},
$$

and $\mathbf{G}$ is a $d \times d$ diagonal matrix containing the angledependent antenna response, propagation gain and initial phase offset of each source.

Without considering this structure, $\mathbf{M}$ may also reflect the imperfections of the array such as calibration errors, antenna coupling effects or inaccuracies in the position of the elements, and propagation effects such as short-delay multipath (scattering in the vicinity of the receiver array). As mentioned, for the purpose of source separation we do not consider this structure and only assume the matrix $\mathbf{M}$ to be left-invertible (this implies $m \geq d$ ).

Our aim is to compute beamformers $\mathbf{w}_{i}, i=1, \cdots, d$, such that $\mathbf{w}_{i}^{H} \mathbf{x}[n]=\hat{s}_{i}[n]$ is an estimate of the $i$-th SSR signal. In this blind source separation context, we can only try to ensure that each $\hat{s}_{i}[n]$ looks like an SSR signal (i.e., that certain properties are satisfied), and that the collection of signal estimates is independent.

\section{Preprocessing}

In our application, $\mathbf{M}$ is typically tall and full column rank, but not square. To simplify our algorithms, we assume that first a (standard) preprocessing is applied on $\mathbf{X}$ to reduce its row dimension from $m$ to $d$. This is done by computing a singular value decomposition of $\mathbf{X}$,

$$
\mathbf{X}=\mathbf{U} \boldsymbol{\Sigma} \mathbf{V}^{H}
$$

where $\mathbf{U}$ and $\mathbf{V}$ are unitary and $\boldsymbol{\Sigma}$ is diagonal containing the singular values in decreasing order. The number of signals $d$ is detected from $\Sigma$ using standard rank detection tests, e.g., based on likelihood ratios [15] or information theoretic criteria such as AIC and MDL [16], [17], [18]. Let $\hat{\boldsymbol{\Sigma}}$ be a diagonal matrix containing the $d$ largest singular values, and $\hat{\mathbf{U}}$ be an $m \times d$ dimensional matrix containing the corresponding columns of $\mathbf{U}$, and define

U, and define the $\tilde{\mathrm{B}}$

$\tilde{\mathrm{B}}_{d}$ aqeording to the model

$$
\mathbf{X}^{\prime}=\left(\hat{\boldsymbol{\Sigma}}^{-1} \hat{\mathbf{U}}^{H} \mathbf{M}\right) \mathbf{S}+\left(\hat{\boldsymbol{\Sigma}}^{-1} \hat{\mathbf{U}}^{H} \mathbf{E}\right) \stackrel{\text { def }}{=} \mathbf{M}^{\prime} \mathbf{S}+\mathbf{E}^{\prime} .
$$

This is the same model as we had before, except that $\mathbf{X}^{\prime}$ is $d \times N$ and $\mathbf{M}^{\prime}$ is $d \times d$ and invertible. In the algorithms, we assume that this preprocessing has been done, and we drop the primes from the notation. The computational complexity (number of multiplications) is of order $m^{2} N$.

The reduction in the number of rows in $\mathbf{X}$ and $\mathbf{M}$ is necessary to avoid the existence of nullspace beamformers $\mathbf{w}_{0}$ such that $\mathbf{w}_{0}^{H} \mathbf{M}=0$. Indeed, such beamformers could be added to a valid separating beamformer $\mathbf{w}_{i}$ without changing the output signal, and only change the output noise. Hence they would destroy the uniqueness of the solution, and complicate the estimation algorithms.

The data covariance whitening implied by premultiplying with $\hat{\boldsymbol{\Sigma}}^{-1}$ is not as essential, but has been applied in similar algorithms because it causes the beamformers to converge asymptotically in $N$ to the Wiener beamformer [19]. Wiener beamformers are attractive because they optimize the output Signal to Interference and Noise Ratio (SINR). Note that after the prewhitening step, the noise $\mathbf{E}^{\prime}$ is no longer spatially white.

\section{Joint diagonalization problem}

The algorithms to be proposed in the next sections lead to joint diagonalization problems. To avoid being repetitious, that problem is presented here in a more general setting.

Let $\mathbf{A}$ be a square matrix. Its eigenvalue decomposition (if it exists, i.e., if $\mathbf{A}$ is regular) is a factorization $\mathbf{A}=\mathbf{V} \mathbf{\Lambda} \mathbf{V}^{-1}$, where $\boldsymbol{\Lambda}$ is a diagonal matrix containing the eigenvalues $\lambda_{i}$, and $\mathbf{V}$ is an invertible matrix containing the eigenvectors $\mathbf{v}_{i}$ as its columns. These are the solutions to the equation $\mathbf{A v}=\mathbf{v} \lambda$. 
Numerically, the eigenvalue problem is often replaced by the Schur decomposition, which is the factorization [20]

$$
\mathbf{A}=\mathbf{Q R Q}^{H}
$$

where $\mathbf{Q}$ is unitary and $\mathbf{R}$ is upper triangular. The diagonal entries of $\mathbf{R}$ are the eigenvalues. It can be obtained by introducing the $\mathrm{QR}$ factorization of $\mathbf{V}$, with the advantage that the Schur decomposition always exists whereas the eigenvalue decomposition does not.

Similarly, the generalized eigenvalue problem (or matrix pencil problem) for a pair of square matrices $(\mathbf{A}, \mathbf{B})$ is to find solutions to $\mathbf{A v}=\mathbf{B v} \lambda$, or the factorization $\mathbf{A V}=\mathbf{B V} \mathbf{\Lambda}$. If $\mathbf{B}$ is invertible, this is the same as computing the eigenvalue decomposition of $\mathbf{B}^{-1} \mathbf{A}$. It is convenient to write this as a joint decomposition. By introducing a matrix $\mathbf{F}$ and $\mathbf{W}=$ $\mathbf{V}^{-1}$, and two diagonal matrices $\boldsymbol{\Lambda}_{A}, \boldsymbol{\Lambda}_{B}$, we obtain

$$
\mathbf{A}=\mathbf{F} \boldsymbol{\Lambda}_{A} \mathbf{W}^{H}, \quad \mathbf{B}=\mathbf{F} \boldsymbol{\Lambda}_{B} \mathbf{W}^{H} .
$$

The generalized eigenvalues are $\boldsymbol{\Lambda}=\boldsymbol{\Lambda}_{B}^{-1} \boldsymbol{\Lambda}_{A}$, but the added generality allows to handle cases where $\mathbf{A}$ and/or $\mathbf{B}$ are singular, or some diagonal entries of $\boldsymbol{\Lambda}_{A}$ and/or $\boldsymbol{\Lambda}_{B}$ are zero. The corresponding Generalized Schur Decomposition is

$$
\mathbf{A}=\mathbf{Q} \mathbf{R}_{A} \mathbf{Z}^{H}, \quad \mathbf{B}=\mathbf{Q} \mathbf{R}_{B} \mathbf{Z}^{H} .
$$

where $\mathbf{Q}$ and $\mathbf{Z}$ are unitary, and $\mathbf{R}_{A}, \mathbf{R}_{B}$ are upper triangular. This decomposition always exists and can be computed iteratively, e.g., using the QZ algorithm, Jacobi iterations, etc. [20]. The diagonal entries of $\mathbf{R}_{A}, \mathbf{R}_{B}$ are those of $\boldsymbol{\Lambda}_{A}, \boldsymbol{\Lambda}_{B}$.

The joint diagonalization problem is a further generalization of the above to more than two matrices:

$$
\mathbf{A}_{i}=\mathbf{F} \mathbf{\Lambda}_{i} \mathbf{W}^{H}, \quad i=1, \cdots, K .
$$

For $K>2$ the problem is overdetermined, hence solutions in general do not exist. However, for a set of matrices that on the basis of model assumptions is expected to admit this factorization, we can try to compute the matrix pair $(\mathbf{F}, \mathbf{W})$ that best diagonalizes the set of given matrices, usually in some least squares sense. It can be computed using generalizations of the QZ algorithm, Jacobi iterations [21], [22], [11], [23], [24], Alternating Least Squares [25], [26] or subspace fitting techniques [27].

The problem can be further generalized by considering rectangular matrices $\mathbf{A}_{i}$, as in this paper. In particular the Alternating Least Squares algorithms are readily generalized to handle this situation. For $K$ matrices of size $m \times n$, the computational complexity is of order $K\left(m^{2} n+m n^{2}\right)$. Alternatively, assuming $m>n$ (tall matrices) and $\mathbf{F}$ to be of full column rank $n$, the problem for rectangular matrices can be reduced to a joint diagonalization of square matrices as follows. Construct $\mathbf{A}=\left[\mathbf{A}_{1}, \cdots, \mathbf{A}_{K}\right]$ and use an SVD to estimate the common column space, which is equal to the column space of $\mathbf{F}$. Let $\mathbf{U}$ be a matrix containing an orthogonal basis ( $n$ columns), and define $\mathbf{A}_{i}^{\prime}=\mathbf{U}^{H} \mathbf{A}_{i}$. Then $\mathbf{A}_{i}^{\prime}=\mathbf{F}^{\prime} \mathbf{\Lambda}_{i} \mathbf{W}^{H}$, with $\mathbf{F}^{\prime}=\mathbf{U}^{H} \mathbf{F}$. The $\mathbf{A}_{i}^{\prime}$ are square $(n \times n)$ and satisfy a joint diagonalization model, and the standard algorithms apply.

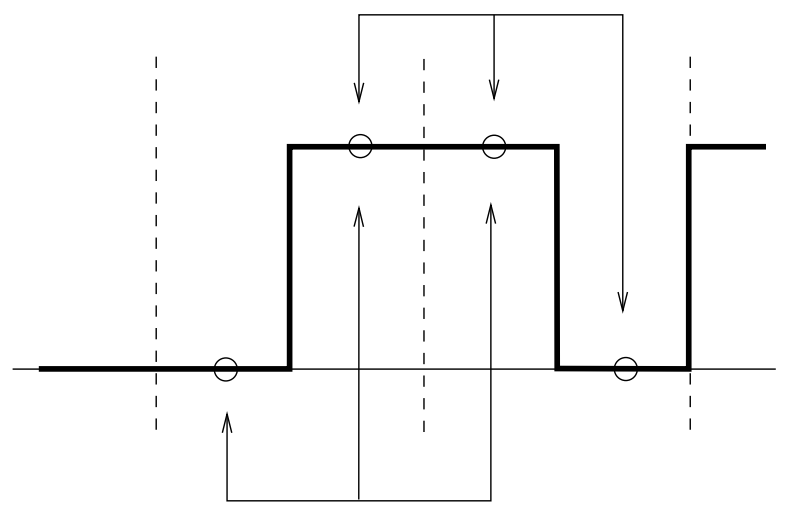

Fig. 1. Manchester Encoding Property: the cross-product of three consecutive $T_{s}$-spaced samples is always equal to zero.

\section{SSR SIGNAL PROPERTIES}

The model of a single SSR reply signal (3) gives rise to several algebraic properties that will be used for blind source separation in Section IV.

\section{A. Encoding properties}

The Manchester encoding of the SSR signals gives rise to an interesting temporal correlation property which is deterministic and independent of the actual transmitted data. If we multiply a sample from the first phase of the Manchester symbol by a sample from the second phase, the result will always be equal to zero. More generally, if we are unsynchronized to the beginning of a Manchester symbol, we can multiply three consecutive $T_{s}$-spaced samples and observe that the result is always zero (see Figure 1). A similar property holds for a single baseband signal $s[n]$ at the receiver, independent of a fractional sampling offset and of the residual carrier frequency.

Property 3.1: Independent of the transmitted data, a received Mode $\mathrm{S}$ reply signal $s[n]$ of the form (3) obeys:

$$
s[n-1] s[n] s[n+1]=0, \quad \forall n \in \mathbb{N}
$$

This property will be used to design a receiver algorithm to separate multiple SSR signals.

\section{B. Zero/Constant Modulus property}

Due to the residual frequency, the received signal samples $s[n]$ are not binary as transmitted, but lie either on the unit circle, or are equal to zero. Moreover, if two subsequent received samples are non-zero, then these samples are related by a factor $\phi=\exp \left(2 \pi \jmath f T_{s}\right)$. These observations lead to the following two properties:

Property 3.2: Static property: $s[n]$ is a Zero-Constant Modulus (ZCM) source if

$$
s[n]=0 \quad \text { or } \quad|s[n]|=1, \quad \forall n \in \mathbb{N}
$$

This is equivalent to

$$
s[n] s^{*}[n] s[n]=s[n]
$$

Property 3.3: Dynamic property: For any integer $k$,

$$
s[n] s^{*}[n-k]=0 \quad \text { or } \quad s[n] s^{*}[n-k]=\phi^{k}, \quad \forall n \in \mathbb{N}
$$


This is equivalent to

$$
s[n] s^{*}[n-k] s[n] s^{*}[n-k]=\phi^{k} s[n] s^{*}[n-k], \quad \forall n \in \mathbb{N}
$$

Note that the mentioned properties also hold for the rows of the source matrix $\mathbf{S}$ as defined in (5).

\section{SEPARATION ALGORITHMS}

The properties presented in Section III are used to derive three algorithms: AZCMA, MDA, and MS-ZCMA. ${ }^{1}$

\section{A. AZCMA: Algebraic Zero-Constant Modulus Algorithm}

The following algorithm was originally derived by Van der Veen and Tol in [12] and is included here for reference. The algorithm is derived for noise-free data (but will of course be applied to noisy data). We consider Property 3.2, and substitute $\mathbf{w}^{H} \mathbf{x}[n]=s[n]$. This shows that $\mathbf{w}$ is a beamformer which returns a ZCM signal if and only if

$$
\mathbf{w}^{H} \mathbf{x}[n] \mathbf{x}[n]^{H} \mathbf{w w}^{H} \mathbf{x}[n]=\mathbf{w}^{H} \mathbf{x}[n], \quad \forall n=1, \cdots, N .
$$

Using properties of Kronecker products, we can separate the unknown $\mathbf{w}$ from the known $\mathbf{x}[n]$ 's. Note that the left-hand side contains only third-order terms of the entries of $\mathbf{w}$, whereas the right-hand side only has first-order terms. This imbalance is overcome by defining

$$
\alpha=\|\mathbf{w}\|^{2}=\mathbf{w}^{H} \mathbf{w}
$$

(which is constant for each w) and multiplying the right-hand side by $1=\frac{1}{\alpha} \mathbf{w}^{H} \mathbf{w}$. This gives

$$
\begin{gathered}
\mathbf{w}^{H} \mathbf{x}[n] \mathbf{x}[n]^{H} \mathbf{w}^{H} \mathbf{x}[n]=\frac{1}{\alpha} \mathbf{w}^{H} \mathbf{w w}^{H} \mathbf{x}[n] \quad \forall n \\
\Leftrightarrow \quad\left(\mathbf{x}^{*}[n] \otimes \mathbf{x}[n] \otimes \mathbf{x}^{*}[n]\right)^{H}\left(\mathbf{w}^{*} \otimes \mathbf{w} \otimes \mathbf{w}^{*}\right)= \\
=\frac{1}{\alpha} \operatorname{vec}\left(\mathbf{I}_{d} \otimes \mathbf{x}^{*}[n]\right)^{H}\left(\mathbf{w}^{*} \otimes \mathbf{w} \otimes \mathbf{w}^{*}\right) \quad \forall n,
\end{gathered}
$$

where $\otimes$ is the Kronecker product. Define matrices $\mathbf{P}_{1}, \mathbf{P}_{2}$ with rows $\left(\mathbf{x}^{*}[n] \otimes \mathbf{x}[n] \otimes \mathbf{x}^{*}[n]\right)^{H}$ and $\operatorname{vec}\left(\mathbf{I}_{d} \otimes \mathbf{x}^{*}[n]\right)^{H}$, respectively. Then the ZCM separation problem is seen to be equivalent to finding all solutions $(\alpha, \mathbf{y}), \alpha \neq 0$ to

$$
\alpha \mathbf{P}_{1} \mathbf{y}=\mathbf{P}_{2} \mathbf{y}, \quad \text { where } \mathbf{y}=\mathbf{w}^{*} \otimes \mathbf{w} \otimes \mathbf{w}^{*} .
$$

To ensure an overdetermined system of equations, we require $\mathbf{P}_{1}$ and $\mathbf{P}_{2}$ to be "tall", i.e., $N \geq d^{3}$. Equation (13) is then a rectangular matrix pencil problem of the form $\mathbf{A x}=\lambda \mathbf{B x}$. The pencil is "singular", i.e., $\mathbf{P}_{1}$ and $\mathbf{P}_{2}$ are not full rank, because the structure of the rows of $\mathbf{P}_{1}$ implies that some of its columns are repeated. This causes additional null space solutions that need to be avoided. Similarly, $\mathbf{y}=\mathbf{w}^{*} \otimes \mathbf{w} \otimes \mathbf{w}^{*}$ has repeated entries, and we want our solutions to satisfy this structure. Because it is known which entries are repeated, it is straightforward to remove the duplicate entries in both $\mathbf{y}$ and the corresponding columns of $\mathbf{P}_{1}$ and $\mathbf{P}_{2}$ by defining a selection matrix $\mathbf{J}$ of size $d^{3} \times \frac{1}{2} d^{2}(d+1)$, such that

$$
\mathbf{y}=\mathbf{J}_{\mathbf{y}}^{\prime}
$$

${ }^{1}$ Matlab implementations of these algorithms are available from the authors upon request. where $\mathbf{y}^{\prime}$ generically has no repeated entries. Set

$$
\mathbf{P}_{1}^{\prime}=\mathbf{P}_{1} \mathbf{J}, \quad \mathbf{P}_{2}^{\prime}=\mathbf{P}_{2} \mathbf{J},
$$

then generically $\mathbf{P}_{1}^{\prime}$ has no repeated columns and has full column rank. ${ }^{2}$ At this point, the pencil problem is replaced by

$$
\alpha \mathbf{P}_{1}^{\prime} \mathbf{y}^{\prime}=\mathbf{P}_{2}^{\prime} \mathbf{y}^{\prime}
$$

where we will assume from now on that $\mathbf{P}_{1}^{\prime}$ has full column rank. Note that by construction $\mathbf{P}_{2}^{\prime}$ has only $d$ nonzero columns. Hence, there are at most $d$ nonzero solutions $\left\{\alpha_{i}\right\}$ to (16). On the other hand, for $d$ SSR signals we know that there are precisely $d$ beamformers $\mathbf{w}_{i}$, so that there are at least $d$ nonzero solutions. It follows that the pair $\left(\mathbf{P}_{2}^{\prime}, \mathbf{P}_{1}^{\prime}\right)$ has precisely $d$ generalized eigenvalues, necessarily equal to $\alpha_{i}=\left\|\mathbf{w}_{i}\right\|^{2}$; the other eigenvalues are 0 . The corresponding eigenvectors $\mathbf{y}_{i}^{\prime}$ are transformed to $\mathbf{y}_{i}=\mathbf{J} \mathbf{y}_{i}^{\prime}$ to add back the repeated entries.

At this point there are two cases. If there are no repeated nonzero eigenvalues, then the $\mathbf{y}_{i}$ are (up to an arbitrary scaling) equal to $\mathbf{y}_{i}=\mathbf{w}^{*}{ }_{i} \otimes \mathbf{w}_{i} \otimes \mathbf{w}^{*}{ }_{i}$, from which $\mathbf{w}_{i}$ is immediately obtained, up to scaling. The correct scaling of $\mathbf{w}_{i}$ follows from the corresponding eigenvalue $\alpha_{i}$. Alternatively, if some of the eigenvalues are repeated, then the corresponding eigenvectors form an arbitrary basis of a subspace which contains the vectors we are looking for. We need to find the correct linear combinations such that the Kronecker structure holds: this is a joint diagonalization problem as shown below.

In fact, the prewhitening step as described in Section II-C has led to a data matrix $\mathbf{X}^{\prime}$ with orthonormal rows. In this case, $\alpha_{i}=\mathbf{w}_{i}^{H} \mathbf{w}_{i}=\mathbf{s}_{i}^{H} \mathbf{s}_{i}$, so that there are repeated eigenvalues whenever two signals have an equal number of nonzero entries, i.e., nearly always. We propose to avoid the detection of equal eigenvalues and apply the joint diagonalization step to the full collection of $d$ eigenvectors of the pencil (16), as follows.

Each eigenvector $\mathbf{y}_{i}$ of size $d^{3}$ is a linear combination of the solutions, or $\mathbf{y}_{i}=\sum_{j=1}^{d} \lambda_{i j} \mathbf{w}^{*}{ }_{j} \otimes \mathbf{w}_{j} \otimes \mathbf{w}^{*}{ }_{j}$, for $i=1, \cdots, d$. If we reshape a single vector $\lambda_{i j}\left(\mathbf{w}^{*}{ }_{j} \otimes \mathbf{w}_{j} \otimes \mathbf{w}^{*}{ }_{j}\right)$ into a $d^{2} \times d$ matrix, we obtain the rank-1 matrix $\left(\mathbf{w}_{j} \otimes \mathbf{w}^{*}{ }_{j}\right) \lambda_{i j} \mathbf{w}_{j}^{H}$. Similarly, reshaping $\mathbf{y}_{i}$ into a $d^{2} \times d$ matrix $\mathbf{Y}_{i}$, we obtain

$$
\mathbf{Y}_{i}=\mathbf{F} \boldsymbol{\Lambda}_{i} \mathbf{W}^{H}
$$

where $\mathbf{W}=\left[\mathbf{w}_{1}, \cdots, \mathbf{w}_{d}\right], \mathbf{F}=\left[\mathbf{w}_{1} \otimes \mathbf{w}^{*}{ }_{1}, \cdots, \mathbf{w}_{d} \otimes \mathbf{w}^{*}{ }_{d}\right]$, and $\boldsymbol{\Lambda}_{i}$ is a diagonal matrix containing the coefficients $\lambda_{i j}$.

Ignoring the structure of $\mathbf{F}$, the problem to obtain $\mathbf{W}$ and the $\left\{\boldsymbol{\Lambda}_{i}\right\}$ from (17) is recognized as a joint diagonalization problem, generalized to rectangular matrices. Thus, the algorithms mentioned in Section II-D can be applied.

With noise, we follow the same algorithm. With $N \geq d^{3}$, the pencil problem (16) is overdetermined. The usual reduction to a pencil with square matrices, $\left(\mathbf{P}_{1}^{\prime H} \mathbf{P}_{2}^{\prime}, \mathbf{P}_{1}^{\prime H} \mathbf{P}_{1}^{\prime}\right)$, amounts to a projection of the column span of $\mathbf{P}_{2}^{\prime}$ onto that of $\mathbf{P}_{1}^{\prime}$, and is a form of noise mitigation. The square pencil has $d^{3}$ eigenvalues,

\footnotetext{
${ }^{2}$ Specific situations still lead to $\mathbf{P}_{1}^{\prime}$ being singular, e.g., if two signals are purely constant modulus, or if two sources share exactly the same frequency. Such pencils can be analyzed using more advanced techniques (see e.g., [28]), but for the sake of simplicity, we will not consider them here. If such a situation arises, the algorithm will fail.
} 
of which we keep the $d$ largest (the others are close to 0 ). The corresponding eigenvectors $\left\{\mathbf{y}_{i}^{\prime}\right\}$ are used in the joint diagonalization step. This by itself is also an overdetermined problem, hence provides additional noise mitigation.

The computational cost of the algorithm is determined by the generalized eigenvalue decomposition of the pair $\left(\mathbf{P}_{1}^{\prime H} \mathbf{P}_{2}^{\prime}, \mathbf{P}_{1}^{\prime H} \mathbf{P}_{1}^{\prime}\right)$. The algorithm is summarized in figure 2. The complexity is of order $N d^{6}$ multiplications.

\section{B. MDA: Manchester Decoding Algorithm}

The Manchester Encoding Property 3.1 can be used to design a receiver algorithm to separate multiple SSR signals. Indeed, if we consider a beamformer $\mathbf{w}$ such that $\hat{s}_{i}[n]=$ $\mathbf{w}^{H} \mathbf{x}[n]$ satisfies equation (9), we obtain

$$
[\mathbf{x}[n+1] \otimes \mathbf{x}[n] \otimes \mathbf{x}[n-1]]^{H}(\mathbf{w} \otimes \mathbf{w} \otimes \mathbf{w})=0
$$

for $n=2, \cdots, N-2$. To collect these conditions, define the matrix $\mathbf{P}:(N-2) \times d^{3}$ as the stack of rows $[\mathbf{x}[n+1] \otimes \mathbf{x}[n] \otimes \mathbf{x}[n-1]]^{H}$ for $n=2, \cdots, N-1$, so that

$$
\mathbf{P} \mathbf{w}^{\diamond}=0, \quad \mathbf{w} \stackrel{\text { def }}{=} \mathbf{w} \otimes \mathbf{w} \otimes \mathbf{w} .
$$

Unlike the case in the previous algorithm, it is not necessary to reduce the dimension of $\mathbf{w}^{\diamond}$ as the rows of the matrix $\mathbf{P}$ do not have redundant entries. On the other hand, operations similar to (14)-(15) may improve the estimation and help to force some structure in the solutions $\mathbf{y}_{i}$.

The rest of the algorithm is quite similar to the one in Section IV-A. For $d$ sources, there are $d$ linearly independent separating beamformers $\mathbf{w}_{i}, i=1, \cdots, d$, as shown in Proposition 4.1 below. Thus we have $d$ linearly independent vectors $\mathbf{w}_{i}^{\diamond}$ that belong to the kernel of $\mathbf{P}$. If the kernel is $d$ dimensional, then the subspace spanned by $\left\{\mathbf{w}_{i}^{\diamond}, i=1, \cdots, d\right\}$ is exactly equal to the kernel of $\mathbf{P}$, and a basis $\left\{\mathbf{y}_{i}\right\}$ for the kernel must be a linear combination of the $\mathbf{w}_{i}^{\diamond}$. Thus, the algorithm is to estimate an arbitrary basis $\left\{\mathbf{y}_{i}\right\}$ for the kernel using the SVD of $\mathbf{P}$, find linear combinations of the basis vectors to map them to the structured vectors $\left\{\mathbf{w}_{i}^{\diamond}\right\}$, and then estimate the corresponding $\mathbf{w}_{i}$ from each vector $\mathbf{w}_{i}^{\diamond}$.

The key step is to find the linear combinations of the basis vectors. This is again a joint diagonalization problem in three dimensions, similar to the case discussed in Section IV-A, and can be solved for the $\mathbf{w}_{i}$ 's.

The algorithm is summarized in figure 3 . The computational cost of the algorithm is determined by the estimation of the kernel of $\mathbf{P}\left((N-2) \times d^{3}\right)$. The complexity is of order $N d^{6}$.

Proposition 4.1: Assume that $\mathbf{M}$ is square and invertible, the sources are statistically stationary and temporally totally overlapping, and that there is no noise. Then for large number of samples $N$, the matrix $\mathbf{P}$ will almost surely have rank $\left(d^{3}-\right.$ $d)$, equivalently its kernel will almost surely be of dimension $d$.

The proof is given in appendix . The proposition implies that for sufficiently large $N$ there are no other solutions than $\left\{\mathbf{w}_{i}^{\diamond}, i=1, \cdots, d\right\}$, so that the problem is identifiable. Experience with similar algorithms indicate that $\mathbf{P}$ is already of maximal rank once it is tall, i.e., $N \geq d^{3}$ is sufficient in practice [29], [30]. This is because it is very unlikely that a random square or tall matrix has a kernel unless there is a structural reason for it. The proposition showed there is no structural reason.

A limitation of the algorithm is that, for completely or almost completely non-overlapping SSR replies, there are additional vectors in the kernel. Indeed, if $\mathbf{w}_{1}$ and $\mathbf{w}_{2}$ are corresponding beamformers, then vectors of the form $\mathbf{w}_{1} \otimes$ $\mathbf{w}_{1} \otimes \mathbf{w}_{2}$ are in the kernel, because they correspond to conditions $s_{1}[n+1] \otimes s_{1}[n] \otimes s_{2}[n-1]=0$, which is always satisfied for non-overlapping source signals. The additional vectors in the kernel will break the assumption on which the algorithm is based (i.e., any vector in the kernel is a linear combination of the $\mathbf{w}_{i}^{\diamond}, i=1, \cdots, d$ ), and without further corrections the algorithm will show poor performance in this situation.

\section{MS-ZCMA: Multi-Shift Zero Constant Modulus Algorithm}

Let $\mathbf{w}$ be a $d$-dimensional beamforming vector to recover $s[n], \mathbf{w}^{H} \mathbf{x}[n]=\hat{s}[n]$. Using properties of the Kronecker product, equation (12) from the ZCM Property 3.3 becomes

$$
\begin{gathered}
\left(\mathbf{x}[n] \otimes \mathbf{x}^{*}[n-\tau] \otimes \mathbf{x}[n] \otimes \mathbf{x}^{*}[n-\tau]\right)^{T}\left(\mathbf{w}^{*} \otimes \mathbf{w} \otimes \mathbf{w}^{*} \otimes \mathbf{w}\right) \\
=\phi^{\tau}\left(\mathbf{x}[n] \otimes \mathbf{x}^{*}[n-\tau]\right)^{T}\left(\mathbf{w}^{*} \otimes \mathbf{w}\right)
\end{gathered}
$$

Let $\mathbf{w}^{\oslash 4}$ be an $(d(d+1) / 2)^{2}$-dimensional vector that contains only the non-redundant elements of the Kronecker product $\left(\mathbf{w}^{*} \otimes \mathbf{w} \otimes \mathbf{w}^{*} \otimes \mathbf{w}\right)$. We define by $\mathbf{J}^{\prime}$ the $d^{4} \times(d(d+1) / 2)^{2}$ matrix such that $\left(\mathbf{w}^{*} \otimes \mathbf{w} \otimes \mathbf{w}^{*} \otimes \mathbf{w}\right)=\mathbf{J}^{\prime} \mathbf{w}^{\oslash 4}$. We also define

$$
\begin{aligned}
\mathbf{w}^{\oslash 2} & =\mathbf{w}^{*} \otimes \mathbf{w} \\
\mathbf{p}_{\tau, n}^{(1)} & =\left(\mathbf{x}[n] \otimes \mathbf{x}^{*}[n-\tau] \otimes \mathbf{x}[n] \otimes \mathbf{x}^{*}[n-\tau]\right)^{T} \mathbf{J}^{\prime} \\
\mathbf{p}_{\tau, n}^{(2)} & =\left(\mathbf{x}[n] \otimes \mathbf{x}^{*}[n-\tau]\right)^{T}
\end{aligned}
$$

Then (20) can be written as

$$
\mathbf{p}_{\tau, n}^{(1)} \mathbf{w}^{\oslash 4}=\phi^{\tau} \mathbf{p}_{\tau, n}^{(2)} \mathbf{w}^{\oslash 2}
$$

Stacking the rows $\mathbf{p}_{\tau, n}^{(1)}$ and $\mathbf{p}_{\tau, n}^{(2)}, n=\tau, \cdots, N$, into matrices $\mathbf{P}_{\tau}^{(1)}$ and $\mathbf{P}_{\tau}^{(2)}$, respectively, we obtain

$$
\mathbf{P}_{\tau}^{(1)} \mathbf{w}^{\oslash 4}=\phi^{\tau} \mathbf{P}_{\tau}^{(2)} \mathbf{w}^{\oslash 2}
$$

where $\mathbf{P}_{\tau}^{(1)}$ is $(N-\tau) \times(d(d+1) / 2)^{2}$, and $\mathbf{P}_{\tau}^{(2)}$ is $(N-\tau) \times d^{2}$. This equation holds for all $\tau \in \mathbb{N}$, and is somewhat similar to the matrix pencil problem (13) considered before, except that the solution vectors on the left and right hand side of the equations are not equal.

Various approaches are possible. As in Section IV-A, we can multiply with $\alpha=\mathbf{w}^{\mathrm{H}} \mathbf{w}$ to reach an equation that involves only $\mathbf{w}^{\oslash 4}$. An second approach is proposed in [12], called AFZA, where the equation is written in terms of a single unknown vector $\left[\begin{array}{ll}\mathbf{w}^{\oslash 4 T} & \mathbf{w}^{\oslash 2 T}\end{array}\right]^{T}$. A third approach which seems to work better is outlined below.

To solve (22), we first need to find the common column span of $\mathbf{P}_{\tau}^{(1)}$ and $\mathbf{P}_{\tau}^{(2)}$. Let $\mathbf{U}_{\tau}$ be a matrix whose columns form an orthonormal basis of this subspace, and let $\mathbf{U}_{\tau}^{(1)}$ and $\mathbf{U}_{\tau}^{(2)}$ be the orthogonal complements of $\mathbf{U}_{\tau}$ over $\mathbf{P}_{\tau}^{(1)}$ and $\mathbf{P}_{\tau}^{(2)}$. Then 
Given a data matrix $\mathbf{X}=[\mathbf{x}[1], \mathbf{x}[2], \cdots, \mathbf{x}[N]]$, compute separating beamformers $\mathbf{W}$ :

1) Detect number of signals $d$ and prewhiten (Section II-C)

$\left(N m^{2}\right)$

2) Construct $\mathbf{P}_{1}^{\prime}$ with rows $\left(\mathbf{x}^{*}[n] \otimes \mathbf{x}[n] \otimes \mathbf{x}^{*}[n]\right)^{H} \mathbf{J}$

and $\mathbf{P}_{2}^{\prime}$ with rows $\operatorname{vec}\left(\mathbf{I}_{d} \otimes \mathbf{x}^{*}[n]\right)^{H} \mathbf{J}, n=1, \cdots, N$

$\left(N d^{3}\right)$

3) Compute $\mathbf{P}_{1}^{\prime H} \mathbf{P}_{2}^{\prime}$ and $\mathbf{P}_{1}^{\prime H} \mathbf{P}_{1}^{\prime}$

4) Solve the matrix pencil $\alpha \mathbf{P}_{1}^{\prime H} \mathbf{P}_{1}^{\prime} \mathbf{y}^{\prime}=\mathbf{P}_{1}^{\prime H} \mathbf{P}_{2}^{\prime} \mathbf{y}^{\prime}$

Let $\left\{\mathbf{y}_{i}^{\prime}\right\}$ be the eigenvectors corresponding to the $d$ largest eigenvalues

5) $\mathbf{y}_{i}=\mathbf{J}_{i}^{\prime}, i=1, \cdots, d$

Reshape $\mathbf{y}_{i}$ into matrices $\mathbf{Y}_{i}\left(\right.$ size $\left.d^{2} \times d\right)$

6) Solve $\mathbf{Y}_{i}=\mathbf{F} \boldsymbol{\Lambda}_{i} \mathbf{W}^{H}(i=1, \cdots, d)$ for $\mathbf{W}$ (Section II-D)

Fig. 2. Summary of AZCMA (in brackets the order of complexity of each step).

Given a data matrix $\mathbf{X}=[\mathbf{x}[1], \mathbf{x}[2], \cdots, \mathbf{x}[N]]$, compute separating beamformers $\mathbf{W}:$
1) Detect number of signals $d$ and prewhiten $($ Section II-C)
2) Construct $\mathbf{P}$ with rows $(\mathbf{x}[n+1] \otimes \mathbf{x}[n] \otimes \mathbf{x}[n-1])^{H}, n=1, \cdots, N$
3) Compute the SVD of $\mathbf{P}\left(\operatorname{size} d^{3} \times d\right)$ and determine a basis $\left\{\mathbf{y}_{i}\right\}$ for the kernel
4) Reshape each $\mathbf{y}_{i}$ into a matrix $\mathbf{Y}_{i}\left(\right.$ size $\left.d^{2} \times d\right), i=1, \cdots d$
5) Solve $\mathbf{Y}_{i}=\mathbf{F} \mathbf{\Lambda}_{i} \mathbf{W}^{H}(i=1, \cdots, d)$ for $\mathbf{W}($ Section II-D)

Fig. 3. Summary of MDA (in brackets the order of complexity of each step).

we can compute the decomposition into 'common' and 'not common' subspaces as

$$
\begin{aligned}
& \mathbf{P}_{\tau}^{(1)}=\left[\begin{array}{ll}
\mathbf{U}_{\tau} & \mathbf{U}_{\tau}^{(1)}
\end{array}\right]\left[\begin{array}{l}
\mathbf{A}_{\tau}^{(1)} \\
\mathbf{B}_{\tau}^{(1)}
\end{array}\right] \\
& \mathbf{P}_{\tau}^{(2)}=\left[\begin{array}{ll}
\mathbf{U}_{\tau} & \mathbf{U}_{\tau}^{(2)}
\end{array}\right]\left[\begin{array}{l}
\mathbf{A}_{\tau}^{(2)} \\
\mathbf{B}_{\tau}^{(2)}
\end{array}\right]
\end{aligned}
$$

where the $\mathbf{B}_{\tau}^{(i)}, i \in\{1,2\}$ are of full row rank. Inserting these two equations into (22), we obtain

$$
\left[\begin{array}{lll}
\mathbf{U}_{\tau} & \mathbf{U}_{\tau}^{(1)} & \mathbf{U}_{\tau}^{(2)}
\end{array}\right]\left[\frac{\frac{\mathbf{A}_{\tau}^{(1)} \mathbf{w}^{\otimes 4}-\phi^{\tau} \mathbf{A}_{\tau}^{(2)} \mathbf{w}^{\oslash 2}}{\mathbf{B}_{\tau}^{(1)} \mathbf{w}^{\otimes 4}}}{-\phi^{\tau} \mathbf{B}_{\tau}^{(2)} \mathbf{w}^{\oslash 2}}\right]=\mathbf{0}
$$

where the first matrix compound has full column rank by definition. Thus, we have

$$
\begin{aligned}
\mathbf{A}_{\tau}^{(1)} \mathbf{w}^{\oslash 4}-\phi^{\tau} \mathbf{A}_{\tau}^{(2)} \mathbf{w}^{\oslash 2} & =\mathbf{0} \\
\mathbf{B}_{\tau}^{(1)} \mathbf{w}^{\oslash 4} & =\mathbf{0} \\
\mathbf{B}_{\tau}^{(2)} \mathbf{w}^{\oslash 2} & =\mathbf{0}
\end{aligned}
$$

Since it is complicated to work with the three equations simultaneously, we propose in our algorithm to use only the last equation (26). This equation holds for any $\tau$, and we can obtain several similar conditions by taking a range of $L$ different $\tau \in \mathbb{N}$. Stacking the matrices $\mathbf{B}_{\tau}^{(2)}$ in a single matrix $\mathbf{Z}$, we obtain

$$
\mathbf{Z} \mathbf{w}^{\ominus 2} \stackrel{\text { def }}{=}\left[\begin{array}{c}
\mathbf{B}_{0}^{(2)} \\
\mathbf{B}_{1}^{(2)} \\
\vdots \\
\mathbf{B}_{L}^{(2)}
\end{array}\right] \mathbf{w}^{\ominus 2}=\mathbf{0}
$$

For $d$ SSR sources, there are $d$ linearly independent beamformers $\mathbf{w}_{i}, i=1, \cdots, d$, and these correspond to $d$ independent solutions: nonzero vectors in the kernel of $\mathbf{Z}$. Note that $\mathbf{Z}$ has $d^{2}$ columns. As with the preceding algorithms, for a sufficient number of time-lags $L$, the matrix becomes very tall and will not have other vectors in the kernel.

Thus, the algorithm continues by estimating an arbitrary orthonormal basis $\left\{\mathbf{y}_{i}\right\}_{i=1}^{d}$ for the kernel of the matrix $\mathbf{Z}$. Similar to the preceding algorithms, each vector $\mathbf{y}_{i}$ of size $d^{2}$ of this basis is a linear combination of the solutions, or $\mathbf{y}_{i}=\sum_{j=1}^{d} \lambda_{i j} \mathbf{w}_{j}^{\ominus 2}$. Reshaping the $\mathbf{y}_{i}$ into $d \times d$ matrices $\mathbf{Y}_{i}$, we obtain $\mathbf{Y}_{i}=\mathbf{W} \boldsymbol{\Lambda}_{i} \mathbf{W}^{H}$, where $\mathbf{W}=\left[\mathbf{w}_{1}, \cdots, \mathbf{w}_{d}\right]$, and the $\boldsymbol{\Lambda}_{i}$ are diagonal matrices containing the coefficients $\lambda_{i j}$. This is the standard joint diagonalization problem, and can be solved for $\mathbf{W}$ as in Section II-D.

The algorithm is summarized in figure 4 . The computational cost is dominated by the decomposition (23) for each $\tau$. This

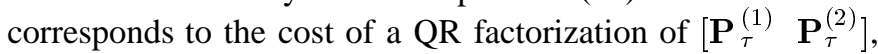
which is of the order $N d^{8}$, therefore the complexity is of order $L N d^{8}$.

The set of time delays $\left\{\tau_{1}, \cdots, \tau_{L}\right\}$ can be chosen arbitrarily, as long as the matrix $\mathbf{Z}$ is expected to achieve its maximal rank. To save some computational cost, we could initially take $L$ small and let it grow until the estimate of the kernel of $\mathbf{Z}$ (updated using subspace tracking algorithms) is considered not to change anymore.

\section{CRAmer-Rao Bound}

The Cramer-Rao Bound (CRB) is a lower bound on the variance of unbiased parameter estimates. We consider here the deterministic CRB, which considers the additive noise as stochastic, whereas the model parameters are regarded as deterministic unknown parameters. ${ }^{3}$ As usual, we consider

\footnotetext{
${ }^{3}$ The bound for this problem was first presented in [31].
} 
Given a data matrix $\mathbf{X}=[\mathbf{x}[1], \mathbf{x}[2], \cdots, \mathbf{x}[N]]$, compute separating beamformers $\mathbf{W}$ :

1) Detect number of signals $d$ and prewhiten (Section II-C)

$\left(N m^{2}\right)$

2) For each delay $\tau$ ( $L$ times)

(a) Construct $\mathbf{P}_{\tau}^{(1)}$ with rows $\left(\mathbf{x}[n] \otimes \mathbf{x}^{*}[n-\tau] \otimes \mathbf{x}[n] \otimes \mathbf{x}^{*}[n-\tau]\right)^{T} \mathbf{J}^{\prime}$ and $\mathbf{P}_{\tau}^{(2)}$ with rows $\left(\mathbf{x}[n] \otimes \mathbf{x}^{*}[n-\tau]\right)^{T}, n=1, \cdots, N$

(b) Estimate $\mathbf{U}_{\tau}$ by subspace intersection of $\mathbf{P}_{\tau}^{(1)}$ and $\mathbf{P}_{\tau}^{(2)}$

(c) Compute $\mathbf{B}_{\tau}^{(2)}$ from $\mathbf{P}_{\tau}^{(2)}$ and $\mathbf{U}_{\tau}$

3) Construct $\mathbf{Z}$ as in eqn. (27) and compute the SVD of $\mathbf{Z}$.

Let $\left\{\mathbf{y}_{i}\right\}$ be the $d$ eigenvectors corresponding to its kernel

4) Reshape eacn $\mathbf{y}_{i}$ into a matrix $\mathbf{Y}_{i}($ size $d \times d)$

Solve $\mathbf{Y}_{i}=\mathbf{W} \boldsymbol{\Lambda}_{i} \mathbf{W}^{H} \quad(i=1, \cdots, d)$ for $\mathbf{W}$ (Section II-D)

Fig. 4. Summary of MS-ZCMA (in brackets the order of complexity of each step).

the noise variance $\sigma^{2}$ as known, since it can be estimated independently of the other parameters. Further, we consider the case of known signals (matrix $\mathbf{B}$ is discrete-valued with entries $\{0,1\}$ and can be assumed as known for the small perturbations of the parameters under which the CRB is valid). In [32], one can find the CRB for a similar problem, which differs only by the absence of the matrix $\mathbf{B}$.

To obtain results which can be compared to DOA estimation algorithms, we consider a calibrated array and write $\mathbf{M}=$ $\mathbf{A}(\boldsymbol{\theta}) \mathbf{G}$, where $\boldsymbol{\theta}=\left[\theta_{1}, \cdots, \theta_{d}\right], \mathbf{A}=\left[\mathbf{a}\left(\theta_{1}\right), \cdots, \mathbf{a}\left(\theta_{d}\right)\right]$, and $\mathbf{a}(\theta)$ is the array response vector for a signal from direction $\theta$. The matrix $\mathbf{G}$ is diagonal, and the diagonal entries contain the complex gains $g_{i}=\rho_{i} e^{j \psi_{i}}$ of the sources as received by the array. The unknown parameters are collected in a vector $\boldsymbol{\lambda}=\left\{\theta_{i}, \rho_{i}, \psi_{i}, f_{i} ; 1 \leq i \leq d\right\}$.

The CRB on the variance of each parameter is given by the corresponding diagonal entries of the inverse of the Fisher Information matrix (FIM) ([33]).

Proposition 5.1: In the case of known signals, the FIM is

where

$$
\operatorname{FIM}_{\alpha}(\boldsymbol{\lambda})=\frac{2}{\sigma^{2}}\left[\begin{array}{cccc}
\mathbf{I}_{\theta \theta} & \mathbf{I}_{\theta f} & \mathbf{I}_{\theta \rho} & \mathbf{I}_{\theta \phi} \\
\mathbf{I}_{f \theta}^{T} & \mathbf{I}_{f f} & \mathbf{I}_{f \rho} & \mathbf{I}_{f \phi} \\
\mathbf{I}_{\rho \theta}^{T} & \mathbf{I}_{\rho f}^{T} & \mathbf{I}_{\rho \rho} & \mathbf{I}_{\rho \phi} \\
\mathbf{I}_{\phi \theta}^{T} & \mathbf{I}_{\phi f}^{T} & \mathbf{I}_{\phi \rho}^{T} & \mathbf{I}_{\phi \phi}
\end{array}\right]
$$

$$
\begin{aligned}
\mathbf{I}_{\theta \theta} & =\operatorname{Re}\left\{\left(\mathbf{G} \mathbf{S ~ S} \mathbf{S}^{H} \mathbf{G}^{H}\right) \odot\left(\mathbf{D}_{A}^{H} \mathbf{D}_{A}\right)^{*}\right\} \\
\mathbf{I}_{f f} & =\operatorname{Re}\left\{\left(\mathbf{G}(\mathbf{C} \odot \mathbf{S})(\mathbf{C} \odot \mathbf{S})^{H} \mathbf{G}^{H}\right) \odot \mathbf{R}_{A}^{*}\right\} \\
\mathbf{I}_{\theta f} & =\operatorname{Re}\left\{\left(\mathbf{G} \mathbf{S}(\mathbf{C} \odot \mathbf{S})^{H} \mathbf{G}^{H}\right) \odot\left(\mathbf{D}_{A}^{H} \mathbf{A}\right)^{*}\right\} \\
\mathbf{I}_{\rho \rho} & =\operatorname{Re}\left\{\left(\mathbf{\Phi} \mathbf{S} \mathbf{S}^{H} \mathbf{\Phi}^{H}\right) \odot\left(\mathbf{A}^{H} \mathbf{A}\right)^{*}\right\} \\
\mathbf{I}_{\theta \rho} & =\operatorname{Re}\left\{\left(\mathbf{G} \mathbf{S ~ S}{ }^{H} \mathbf{\Phi}^{H}\right) \odot\left(\mathbf{D}_{A}^{H} \mathbf{A}\right)^{*}\right\} \\
\mathbf{I}_{f \rho} & =\operatorname{Re}\left\{\left[(\mathbf{G}(\mathbf{C} \odot \mathbf{S}))(\mathbf{\Phi} \mathbf{S})^{H}\right] \odot\left(\mathbf{A}^{H} \mathbf{A}\right)^{*}\right\} \\
\mathbf{I}_{\phi \phi} & =\operatorname{Re}\left\{\left[(\mathbf{G} \mathbf{S})(\mathbf{G} \mathbf{S})^{H}\right] \odot\left(\mathbf{A}^{H} \mathbf{A}\right)^{*}\right\} \\
\mathbf{I}_{\theta \phi} & =\operatorname{Re}\left\{j\left[(\mathbf{G} \mathbf{S})(\mathbf{G} \mathbf{S})^{H}\right] \odot\left(\mathbf{D}_{A}^{H} \mathbf{A}\right)^{*}\right\} \\
\mathbf{I}_{f \phi} & =\operatorname{Re}\left\{j\left[(\mathbf{G}(\mathbf{C} \odot \mathbf{S}))(\mathbf{G} \mathbf{S})^{H}\right] \odot\left(\mathbf{A}^{H} \mathbf{A}\right)^{*}\right\} \\
\mathbf{I}_{\rho \phi} & =\operatorname{Re}\left\{j\left[(\mathbf{\Phi} \mathbf{S})(\mathbf{G} \mathbf{S})^{H}\right] \odot\left(\mathbf{A}^{H} \mathbf{A}\right)^{*}\right\}
\end{aligned}
$$

where $\mathbf{D}_{A}=\left[\frac{\partial \mathbf{a}}{\partial \theta_{1}}\left(\theta_{1}\right), \cdots, \frac{\partial \mathbf{a}}{\partial \theta_{d}}\left(\theta_{d}\right)\right], \boldsymbol{\Phi}$ is a diagonal matrix containing the phases, $e^{j \psi_{i}}, \mathbf{R}_{A}=\mathbf{A}^{H} \mathbf{A}, \mathbf{C}=$ $j 2 \pi T[1, \cdots, 1]^{T}[0,1, \cdots, N-1]$, and $\mathbf{S}=\mathbf{F} \odot \mathbf{B}$.

The proof is straightforward and presented in [31].

\section{Simulations}

To demonstrate the effectiveness of the proposed algorithms (AZCMA, MDA, and MS-ZCMA), we compare them to JADE [21] (a HOS method based on fourth-order statistics), to EF-ICA [34] (a HOS method which forces the statistical independence of the outputs, based on Fast-ICA), to AFZA (one of the other methods proposed in [12]), to ESPRIT [35], and to the Cramer-Rao Bound. ${ }^{4}$

For the simulations, we have considered a calibrated array of $M=4$ elements, with an inter-element distance of a half wavelength and in absence of multipath. The array set-up is chosen to be able to compare to ESPRIT, and to have a simple way to modify the conditioning of the problem by reducing the angle separation between the sources. As source signals, we generated a uniform random sequence of $N=100 \mathrm{SSR}$ samples (50 Manchester encoded symbols with 2 samples per symbol). This is a bit shorter than actual SSR packets, and does not include the training preamble.

Unless specified otherwise, the default simulation parameters are equal powered sources with an SNR of $30 \mathrm{~dB}$ per antenna element, DOAs equal to $\left[70^{\circ}, 110^{\circ}\right]$ measured from array end-fire, no time offsets (completely overlapping data packets), and frequency offsets equal to $\left[-5 \cdot 10^{4}, 5 \cdot 10^{4}\right] \mathrm{Hz}$. We will subsequently vary the SNR, the DOA separation, and the time offset, respectively.

Most statistics are based on 10,000 independent MonteCarlo runs. For MS-ZCMA, we choose the following set of $L=11$ time delays $\tau_{\ell}$, which has shown satisfactory results: $\left\{0,1,-1,2,-2, \frac{N}{10}, \frac{-N}{10}, \frac{N}{4}, \frac{-N}{4}, \frac{N}{2}, \frac{-N}{2}\right\}$.

In this simulation, the computational complexity (number of multiplications) of each of the algorithms is roughly $10^{4}$ flops for AZCMA and MDA, and $10^{6}$ flops for MS-ZCMA.

We show performance first of all in terms of the failure rate, where a failure is declared if we recover the same source twice, rather than two independent sources. A failure is also declared if the output SINR of any source is below $6 \mathrm{~dB}$. For the cases without failure, we estimate the DOA $\theta_{i}$ of each source via

$$
\hat{\theta}_{i}=\arg \max _{\theta}\left\|\mathbf{a}(\theta)^{H} \hat{\mathbf{a}}_{i}\right\|^{2}, \quad i=1, \cdots, d
$$

\footnotetext{
${ }^{4}$ We also compared to SOBI [36], but it did not perform well. We do not present the results so as not to clutter the graphs.
} 
where $\hat{\mathbf{a}}_{i}$ is the $i$-th column of $\hat{\mathbf{A}}=\mathbf{W}^{\dagger}$, the pseudo-inverse of the matrix $\mathbf{W}$. To judge the quality of the beamformers, We show the root mean squared error (RMSE) of the DOA estimates, averaged over both sources. (The two sources have similar RMSEs. Also the behavior of the frequency estimates is quite similar and is not shown for brevity.) We also present the SINR at the output of the beamformer, which is defined as the ratio of the power of the desired source over the combined power of the interference sources plus the noise power.

In the first simulation, we vary the input SNR over the range $[5,30] \mathrm{dB}$. Figure $5(a)$ presents the corresponding failure rates. We observe that JADE fails nearly always. The reason is that JADE is based on exploiting differences in the fourth-order statistics, whereas, for completely overlapping sources, the cumulants of up to order 5 are expected to be small or zero [9], [10]: the sources are "pseudo-Gaussian". EF-ICA includes a non-linear step and therefore implicitly uses also statistics of order above 5 . It could separate the sources, but the failure rate is high (roughly 0.35). An explanation is that a large number of samples (order few thousand) is needed to exploit the statistical independence. AZCMA has a high failure rate for SNRs below 15-20 dB. MDA and AFZA begin to fail for SNRs below 10 $\mathrm{dB}$, while MS-ZCMA and ESPRIT do not fail for SNRs above $5 \mathrm{~dB}$. Figure 5(b) presents the RMSE of the DOA for the cases without failure. For SNRs above $20 \mathrm{~dB}$, AZCMA and AFZA reach a floor of $0.1^{\circ}$ in standard deviation. Note that the MDA, the MS-ZCMA, and the ESPRIT algorithms are quite near the Cramer-Rao Bound and are consistent. The erratic behavior of JADE is partly due to the very small number of non-failed runs. In Figure 5(c), the output SINR is shown as a function of the SNR. The performance of the algorithms are quite similar, except for JADE and EF-ICA. The output SINR is larger than the input SNR by 5 up to nearly $6 \mathrm{~dB}$, which is consistent with the maximum gain of $6 \mathrm{~dB}$ that can be expected with four antennas for a single signal.

In a second simulation, we varied the angle separation between the two sources. Note that, with 4 antennas, the array beamwidth is about $45^{\circ}$. Figure 6(a) shows again that JADE is not reliable, and also EF-ICA has a very high failure rate. For small angle separations (below $6^{\circ}$ ), AZCMA and ESPRIT are the first algorithms to break down. Only MDA, MS-ZCMA and AZFA can handle very closely spaced sources. Figure 6(b) demonstrates that only MDA, MS-ZCMA and ESPRIT can attain the CRB for large angle separation. For all algorithms, there is room for improvement for small angle separations. Failures are caused because in the initial SVD, a signal singular vector will be replaced by a noise singular vector, which will make it impossible to separate the two closely spaced sources. (The same happens if the number of sources is underestimated.) Note that, in the context of our application, it is rare to have small separations because typically the receiver antenna array would be at a different location than the requesting radar beam. In figure 6(c), the output SINR of all algorithms are again quite similar, except for JADE and EF-ICA. The SINR tends to a limit of $36 \mathrm{~dB}$ for large angle separation, which is equal to the initial input $30 \mathrm{~dB}$ plus $6 \mathrm{~dB}$ of antenna gain.

Finally, we simulate a varying time offset between the ar-

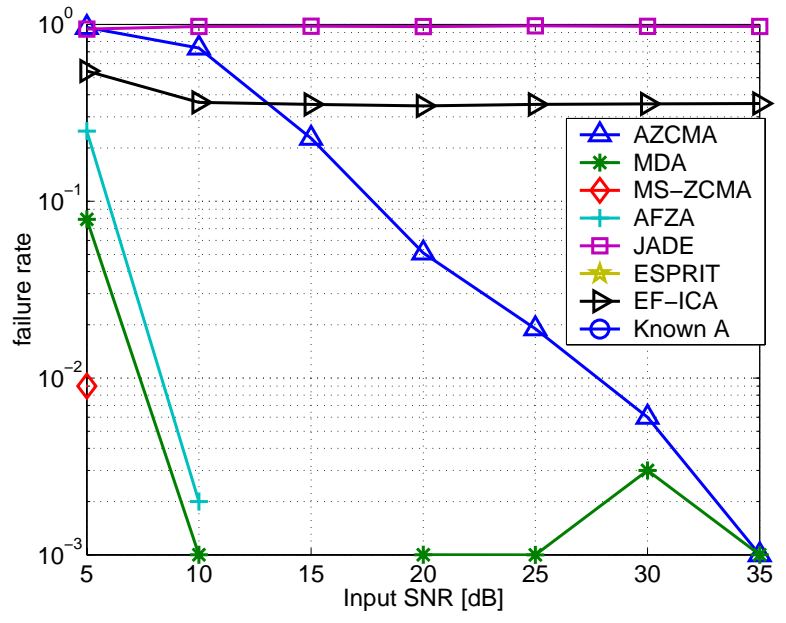

(a)

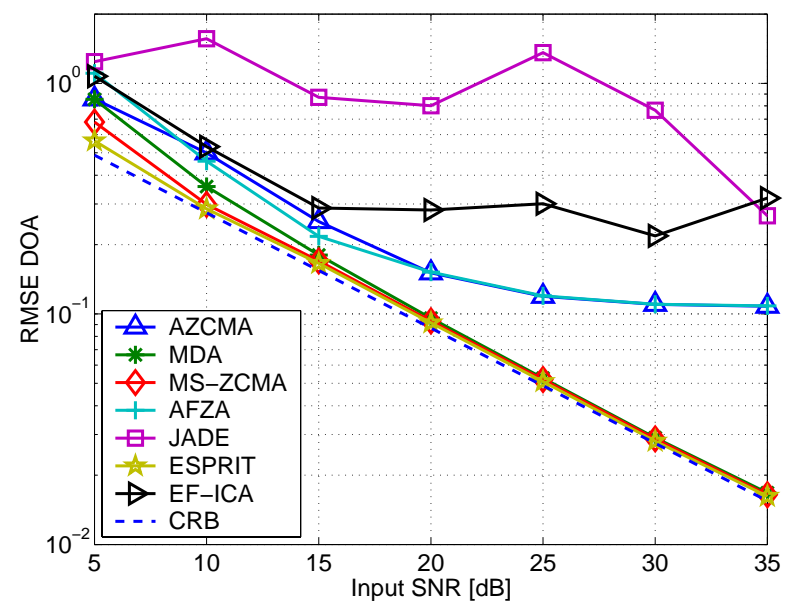

(b)

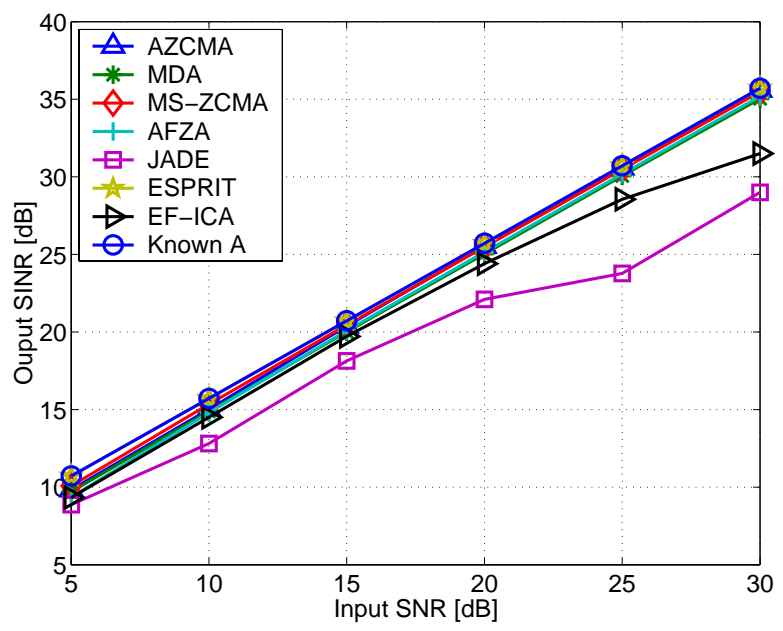

(c)

Fig. 5. Algorithm performance for varying SNR: (a) failure rate, (b) RMSE of the DOA estimates, $(c)$ output SINR. 


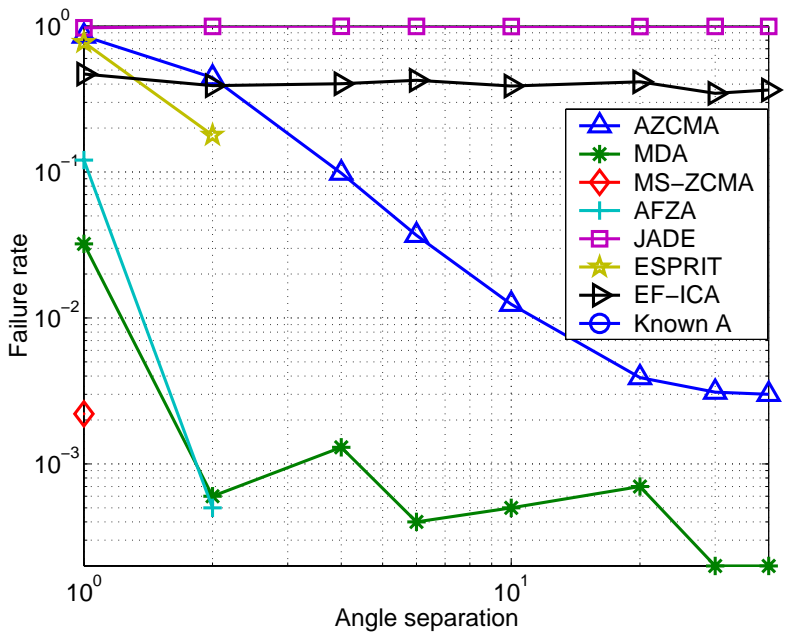

(a)

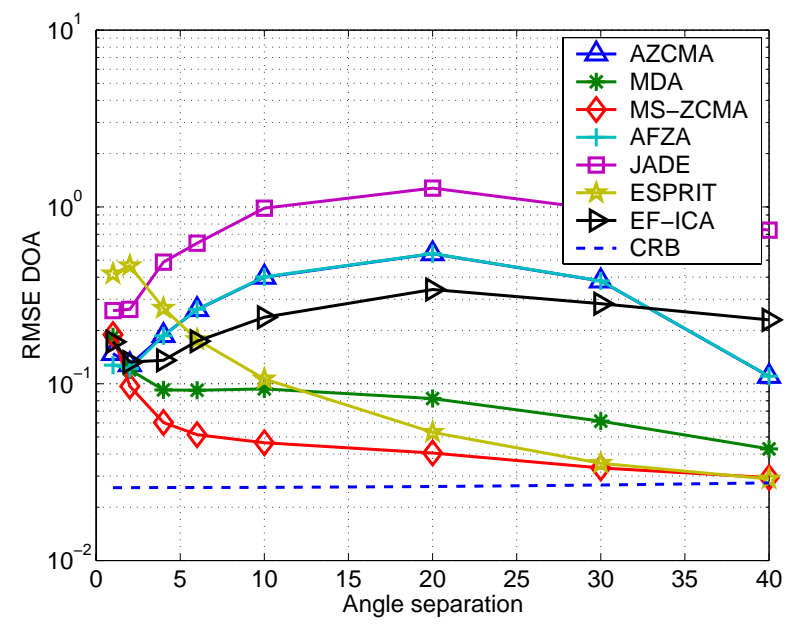

(b)

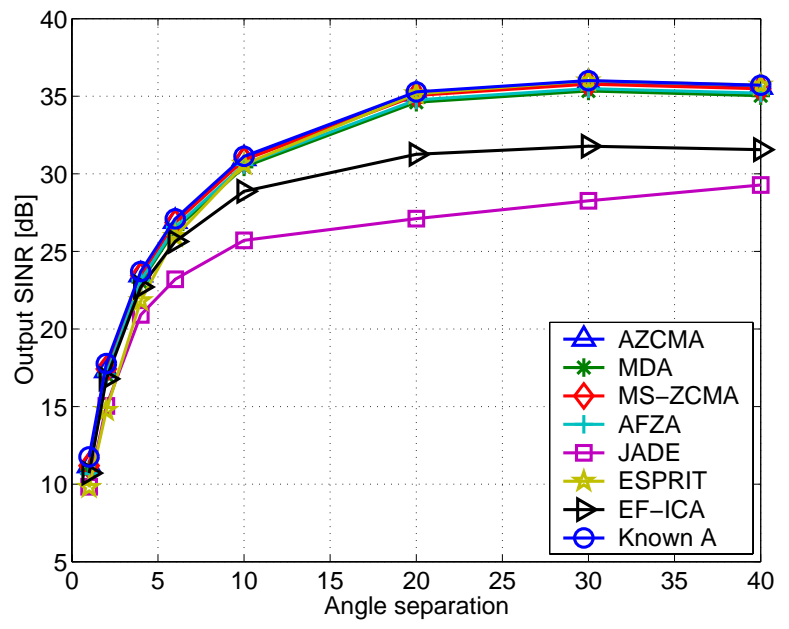

(c)

Fig. 6. Algorithm performance for varying DOA separation: (a) failure rate, (b) RMSE of the DOA estimates, (c) output SINR.

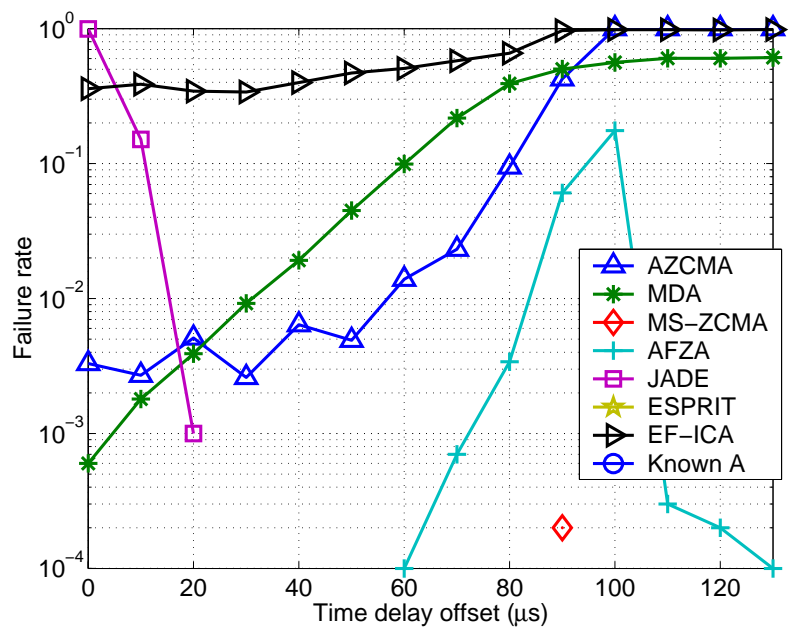

(a)

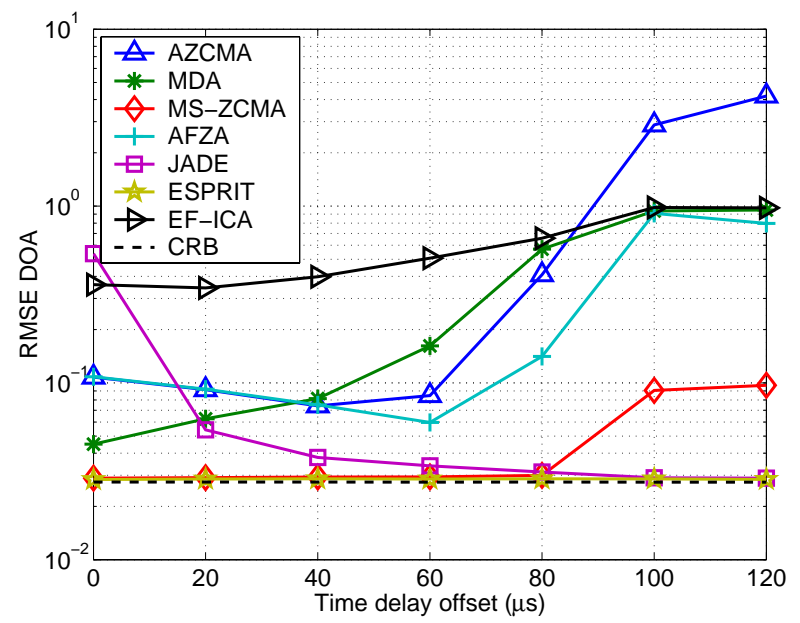

(b)

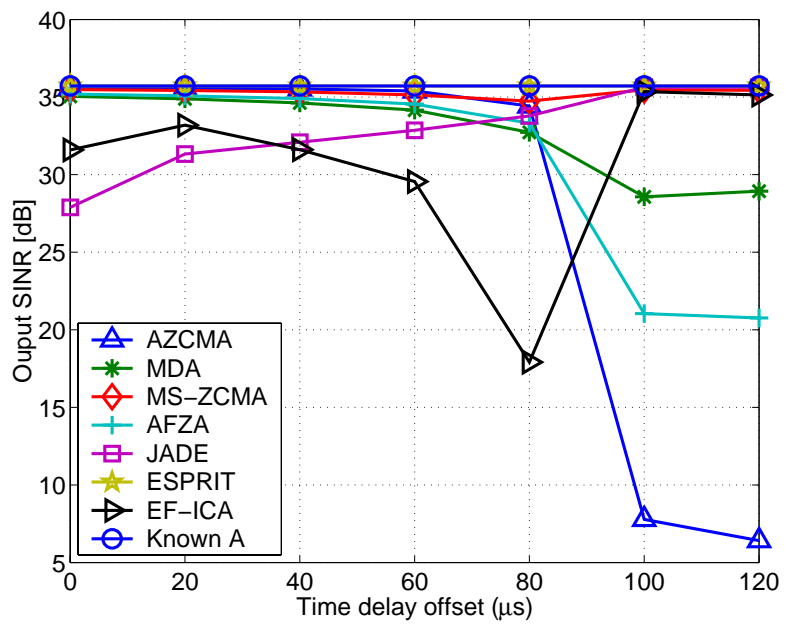

(c)

Fig. 7. Algorithm performance for varying time offset between the two data packets: $(a)$ failure rate, $(b)$ RMSE of the DOA estimates, $(c)$ output SINR 


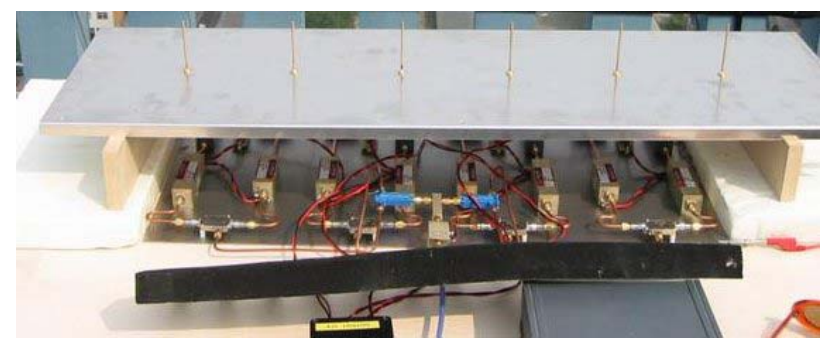

Fig. 8. The experimental system.

rival of the two data packets. Figure $7(a)$ shows that AZCMA and MDA cannot handle well cases of non-overlapping sources, because this leads to additional nullspace solutions in the matrix pencils. On the other hand, as soon as the sources are not completely overlapping, JADE performs well, since the sources are not pseudo-Gaussian anymore. EF-ICA performs worse with increasing time offset until it gives $100 \%$ failure in case of non-overlapping sources. MS-ZCMA and ESPRIT have an acceptable failure rate performance over the range of time delay offsets. Figure 7(b) shows that MS-ZCMA, JADE and ESPRIT have RMSE close to the CRB, with JADE having an error increase for small time offsets and MS-ZCMA for non-overlapping packets. The output SINR presented in figure $7(c)$ confirms that JADE performs better with increasing time delay offset, while the effect is opposite for AZCMA, MDA, and AFZA.

In summary, the simulations show that MS-ZCMA is overall the most reliable algorithm, with the exception of cases with completely non-overlapping packets. AZCMA is not competitive. MDA is a viable alternative (in view of its lower complexity) for small delay offsets only. None of the regular blind source separation algorithms is applicable.

\section{EXPERIMENTAL RESULTS}

\section{A. Experimental setup}

To test the algorithms on real data, we have developed a 4-channel phased array measurement system (see Figure 8). The array consist of 6 linearly equispaced antennas. The two endpoint antennas are "dummy" and loaded with a matching impedance, their presence is to make the coupling between antennas uniform. The four central antennas feed different receiving chains, which down-convert the signal from the radio frequency $(1090 \mathrm{MHz})$ to an intermediate frequency (10 $\mathrm{MHz}$ ), filter around the band of interest and amplify the signal. The four signals are sampled at $50 \mathrm{MS} / \mathrm{s}$ and digitized by a digital oscilloscope which also stores them for later offline processing. The sampling resolution is 8 bits. Offline, the signal is converted to a complex baseband signal using a Hilbert transform, digitally lowpass filtered with a cutoff at an equivalent frequency of $10 \mathrm{MHz}$.

The array has not been calibrated. Measurements indicate that the array response does not follow the ULA response, which may be caused by coupling or multipath, e.g., due to nearby metallic objects. For this reason, it is not possible to evaluate the DOAs, and thus we do not compare to the ESPRIT algorithm. We limit ourselves to source separation in this section. Moreover, we will not further investigate AZFA and EF-ICA as their performances are not competitive.

\section{B. Experimental results}

The array was installed on the roof of our building at TU Delft (approx. $90 \mathrm{~m}$ high, with several large radar dishes and metallic structures nearby), and several data sets were recorded. Most of the recorded data sets have a short time duration, $300 \mu \mathrm{s}$, which is sufficient to contain a Mode-S signal. In these data sets, there were only a few cases of actual overlaps (we are located $50 \mathrm{~km}$ away from the nearest major airport, so the aircraft density is relatively low), but with very good SNR ( 25 to $40 \mathrm{~dB}$ ). We have created artificial data sets by randomly mixing pairs of measured signals at different delay offsets, with the advantage that the true delay offsets are known and that we can easily manipulate the delay offsets, noise power and source powers (i.e., the SNR and SINR). (The array response vectors and frequency offsets cannot be changed.) The mixing is performed simply by adding the two received blocks $\mathbf{X}_{1}$ and $\mathbf{X}_{2}$ over the 4 channels with an additional noise matrix:

$$
\mathbf{X}=\alpha_{1} \mathbf{X}_{1}+\alpha_{2} \mathbf{X}_{2}+\mathbf{E} .
$$

This gave in total 700 combinations to establish the algorithm performance. Since we know the mixing and can easily estimate the "true" array response vectors from the unmixed datasets, we know the true mixing matrix M, see Eq. (4), in each experiment. We use the pseudo-inverse of this matrix to obtain a reference performance.

We present the results from two series of experiments, where we compare the performance of the algorithms to the pseudo-inverse of the true mixing matrix $\mathbf{M}$.

In a first experiment, for every pair of sources, we add them pairwise without time delay offset, and vary the SNR by adding complex Gaussian noise.

Figure 9(a) shows the resulting failure rates as a function of the SNR. Comparing to the simulation (see Figure 5), we see that AZCMA and JADE have similar performance (high failure rates). MDA, MS-ZCMA and "Known M" have somewhat worse but still quite similar failure rates for the measured data. Figure $9(b)$ shows the output SINR as a function of the SNR. It is consistent with the simulations. The performance of all algorithms except JADE is similar to the case where the true mixing matrix is known.

In a second experiment, we use equi-powered sources, add noise to achieve an SNR equal to $20 \mathrm{~dB}$, and only vary the time delay offset. Figure 10 $(a)$ presents the failure rate as a function of the delay of the second source, and 10(b) the output SINR. Compared to the simulations (Fig. 7), AZCMA now also has a high failure rate for small separations. Again, MDA has sufficient performance only for small offsets, and is not reliable for larger offsets. MS-ZCMA has a good performance over the complete range of offsets, and is within $2 \mathrm{~dB}$ of the "known mixing matrix" reference line. JADE requires large time offsets for reliable performance, and in that case gets close to the reference line as well.

In summary, we have seen that the algorithms have similar behavior in simulations as in experiments. The performance 

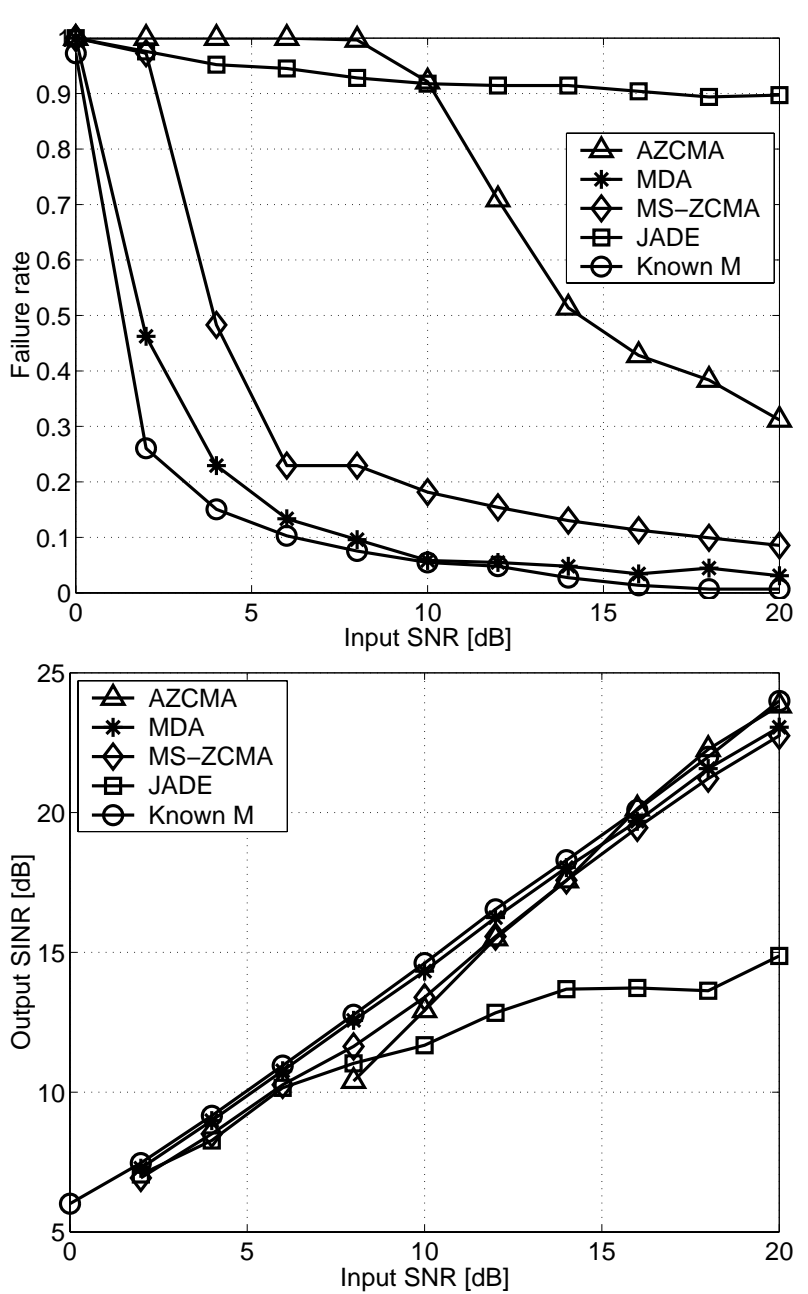

Fig. 9. Performance results using data synthesized from measured singlesource data; varying input SNR: $(a)$ failure rate; $(b)$ output SINR.

of MS-ZCMA is close to the case of a known mixing matrix. MDA is reliable only for small time offsets between the overlapping packets. Conversely, JADE is reliable only for large time offsets. AZCMA is not competitive.

\section{CONCLUSIONS}

We presented three algorithms (AZCMA, MDA and MSZCMA) to separate overlapping SSR replies impinging on an antenna array. Simulations have shown that AZCMA is not reliable, but MDA and MS-ZCMA behave reliable for data packets that are highly overlapping. For small amounts of overlap, only MS-ZCMA is reliable. The proposed algorithms use properties of the sources, hence can work with uncalibrated or non-linear arrays, which is an advantage over algorithms based on the array manifold structure, such as ESPRIT. For completely overlapping data packets, JADE is not applicable because the fourth-order cumulants are expected to be small; indeed its performance is poor. For small amounts of overlap, JADE is functional. We developed an experimental platform, and demonstrated that MS-ZCMA performs quite well on real data as well.

In actual implementations, we recommend that first a test is made to detect if sources are non-overlapping or partially
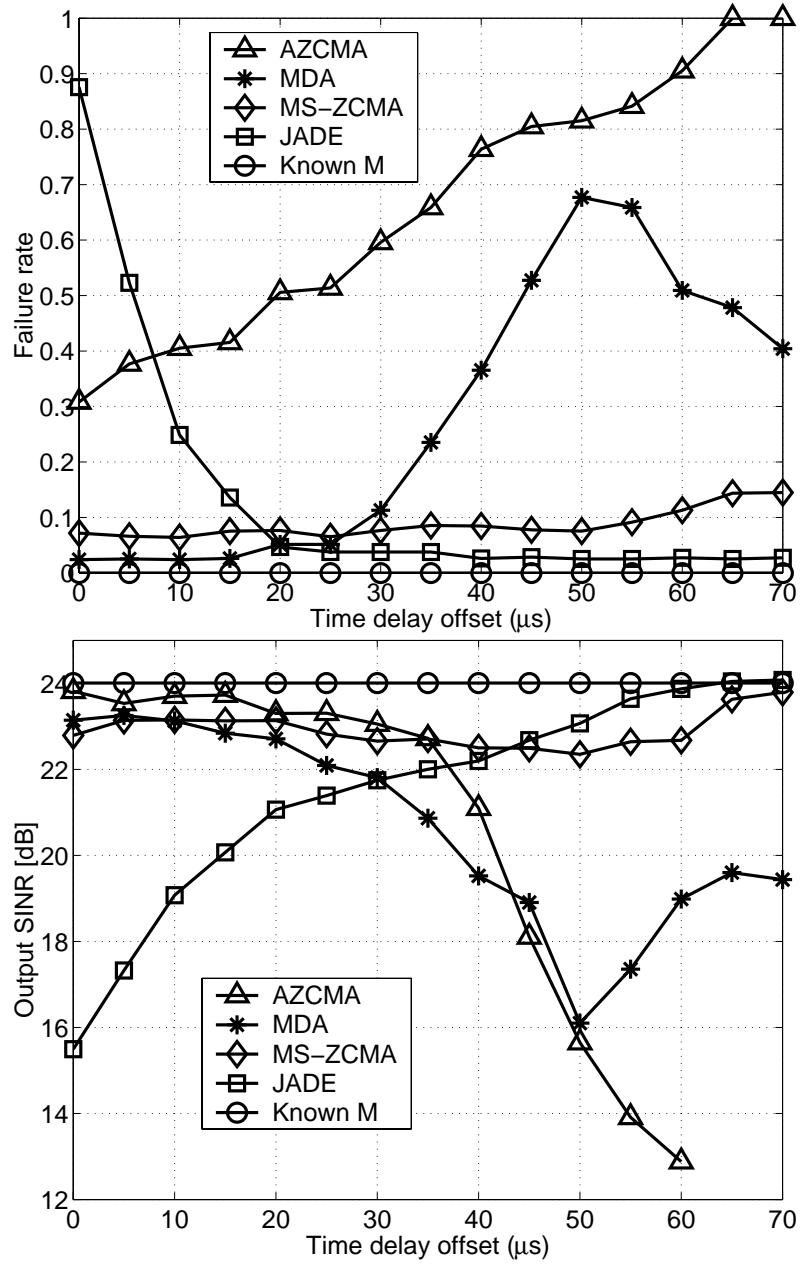

Fig. 10. Performance results using data synthesized from measured singlesource data; varying time offsets between the two sources: $(a)$ failure rate; (b) output SINR.

overlapping. If so, simple algorithms can be used for estimating the beamformers and separating the sources [14]. If it is detected that sources are significantly overlapping, then MSZCMA and MDA can be applied. The complexity of these algorithms is higher, but not prohibitive since the number of simultaneous sources is likely to be small. Together this makes a good candidate for improving the reception of the next generation of Secondary Surveillance Radar.

\section{APPENDIX}

\section{PROOF OF PROPOSITION 4.1}

We need to show that for sufficiently large $N$, the kernel of $\mathbf{P}$ is of dimension $d$, and not larger than $d$. We assume a stationary situation (signals are present for $N \rightarrow \infty$ ), so that we can apply ergodicity. Using the model $\mathbf{x}[k]=\mathbf{M s}[k]$, we have

$\mathbf{x}[k+1] \otimes \mathbf{x}[k] \otimes \mathbf{x}[k-1]=(\mathbf{M} \otimes \mathbf{M} \otimes \mathbf{M})(\mathbf{s}[k+1] \otimes \mathbf{s}[k] \otimes \mathbf{s}[k-1]$

Let $\mathbf{S}_{3} \quad$ be a matrix with rows $\mathbf{s}^{(3) H} \stackrel{\text { def }}{=}$ $[\mathbf{s}[k+1] \otimes \mathbf{s}[k] \otimes \mathbf{s}[k-1]]^{H}$, for $k=2, \cdots, N-1$. 
Then $\mathbf{P}$ can be written as

$$
\mathbf{P}=\mathbf{S}_{3}(\mathbf{M} \otimes \mathbf{M} \otimes \mathbf{M})^{H}
$$

Since $\mathbf{M}$ is invertible, $(\mathbf{M} \otimes \mathbf{M} \otimes \mathbf{M})$ is invertible as well, so that the rank of $\mathbf{P}$ is equal to the rank of $\mathbf{S}_{3}$.

Define the sample correlation matrix corresponding to $\mathbf{S}^{(3)}$ as $\hat{\mathbf{R}}_{s s}^{(3)}=\frac{1}{N} \mathbf{S}_{3}^{H} \mathbf{S}_{3}$. It has the same rank as $\mathbf{S}_{3}$ as soon as $N \geq d^{3}$. We will verify the rank of $\hat{\mathbf{R}}_{s s}^{(3)}$ as $N$ tends to infinity, i.e., when $\hat{\mathbf{R}}_{s s}^{(3)}$ converges to the correlation matrix $\mathbf{R}_{s s}^{(3)}$ of $\mathbf{s}^{(3)}$, under statistical assumptions on the data.

Denote the $k$-th sample of the $m$-th source as $s_{m}[k]=$ $b_{m}[k] \phi_{m}^{k}$, where $b_{m}[k]$ is the transmitted symbol (0 or 1 with equal probability) and $\phi_{m}$ is the residual phase rotation, random on the unit circle. The $i, j$-th entry of $\hat{\mathbf{R}}_{s s}^{(3)}$ is

$$
\begin{aligned}
\left(\hat{\mathbf{R}}_{s s}^{(3)}\right)_{i, j}=\frac{1}{N} \sum_{k=2}^{N-1} \quad & b_{m}[k-1] b_{n}[k] b_{l}[k+1] b_{o}[k-1] b_{p}[k] \\
& \phi_{m}^{-(k-1)} \phi_{n}^{-k} \phi_{l}^{-(k+1)} \phi_{o}^{k-1} \phi_{p}^{k} \phi_{q}^{k+1}
\end{aligned}
$$

where $i=m d^{2}+n d+l$, and $j=o d^{2}+p d+q$, for $m, n, l, o, p, q=1, \cdots, d$. As $N \rightarrow \infty, \hat{\mathbf{R}}_{s s}^{(3)}$ converges to $\mathbf{R}_{s s}^{(3)}$.

Before demonstrating that the rank of $\mathbf{R}_{s s}^{(3)}$ is $d^{3}-d$, we show it in the case $d=2$. Re-order the columns and the rows in order to follow the triplets $(m, n, l)=(1,1,1),(1,1,2)$, $(1,2,1),(2,1,1),(1,2,2),(2,1,2),(2,2,1),(2,2,2)$. Then the matrix converges to a diagonal matrix whose diagonal entries are:

$$
\frac{1}{16}\left[\begin{array}{llllllll}
0 & 1 & 2 & 1 & 1 & 2 & 1 & 0
\end{array}\right]
$$

which is of rank $6=2^{3}-2$. There are precisely $d=2$ columns equal to zero, which gives the kernel a dimension of 2 . The rest of the matrix is block-diagonal with non-singular blocks of size $3 \times 3$.

Now, we consider the general case. For triplets that are not equal up to a permutation, $\{m, n, l\} \neq\{o, p, q\}$, the residual carrier induces the term $\left(\hat{\mathbf{R}}_{s s}^{(3)}\right)_{i, j}$ to tend towards zero. By reordering the rows and columns of $\mathbf{R}_{s s}^{(3)}$, we transform it into a block-diagonal matrix, with three kinds of sub-matrices.

1) The first kind of sub-matrices are of size $1 \times 1$ for triplets of the form $(n, n, n)$, for $n=1, \cdots, d$, with value

$$
\left(\hat{\mathbf{R}}_{s s}^{(3)}\right)_{i, i}=\frac{1}{N} \sum_{k=2}^{N-1} b_{n}^{2}[k-1] b_{n}^{2}[k] b_{n}^{2}[k+1]
$$

where $i=n\left(d^{2}+d+1\right)$. These elements are equal to zero because of the Manchester encoding property 3.1. There are precisely $d$ sub-matrices of this kind. They thus contribute $d$ dimensions to the kernel of $\mathbf{R}_{s s}^{(3)}$.

2) The second kind of sub-matrices are of size $3 \times 3$ for triplets of the form $(n, n, m)$ and $n \neq m$. Denote the corresponding submatrices by $\mathbf{R}_{2}$. Given that any nondiagonal entry of the matrix will contain at least 3 consecutive samples of $b_{n}$, property 3.1 ensures that they are equal to zero. Then the matrix $\mathbf{R}_{2}$ converges to a diagonal matrix whose diagonal entries are:

$$
\mathbf{R}_{2}=\frac{1}{16}\left[\begin{array}{lll}
1 & 2 & 1
\end{array}\right]
$$

which is full rank. There are precisely $d(d-1)$ submatrices of this kind.

3) The third kind of sub-matrices are of size $6 \times 6$ for triplets of forms $\{m, n, l\}$ and $m \neq n \neq l$. Denote by $\mathbf{R}_{3}$ the corresponding sub-matrices. A similar but more tedious analysis reveals that $\mathbf{R}_{3}$ converges to a certain nondiagonal but full-rank matrix. There are precisely $\frac{d(d-1)(d-2)}{6}$ sub-matrices of this kind.

Hence, the rank of $\mathbf{R}_{s s}^{(3)}$ is equal to $d^{3}-d$. Thus, as the number of samples tends towards infinity, $\mathbf{S}_{3}$ and $\mathbf{P}_{3}$ will have rank $\left(d^{3}-d\right)$. By continuity, this will almost surely be the case for finite but sufficiently large $N$.

\section{REFERENCES}

[l] $\mathrm{M}_{1}$ Stevens, Secondary Surveillance Radar. Artech house, Norwood, MA, 1988.

[2] G. Galati, S. Bartolini, and L. Menè, "Analysis of SSR signals by super resolution algorithms," in Proc. IEEE ISSPIT 2004, (Roma, Italy), Dec. 2004.

[3] R. Trim, "Mode S: an introduction and overview," Electronics \& Communication Engineering Journal, vol. 2, pp. 53-59, Apr. 1990.

[4] N. Petrochilos and P. van Genderen, "A new approach to handle SSR replies," in Conférence Radar, (Brest, France), May 1999.

[5] N. Petrochilos, Algorithms for Separation of SSR Replies. PhD thesis, TU Delft, Delft, The Netherlands, Dec. 2002. ISBN 90-407-2371-0, http://cas.et.tudelft.nl/ nicolas.

[6] P. Bezousek, "A passive radar surveillance system VERA for ATC," in IRS'98, (Munich, Germany), 1998.

[7] G. Galati, P. Magarò, M. Gasbarra, and M. Leonardi, "New signal processing techniques in SSR-Mode $\mathrm{S}$ replies multilateration for ASMGCS," in Proc. of IRS 2004, (Warsaw, Poland), 19-21 May 2004.

[8] E. Chaumette, P. Comon, and D. Muller, "An ICA-based technique for radiating sources estimation; application to airport surveillance," IEE Proceedings - Part F, vol. 140, pp. 395-401, Dec. 1993.

[9] N. Petrochilos and P. Comon, "Separation de signaux ZCM: application en radar SSR," in Proc. GRETSI 2003, (Paris, France), Sept. 2003.

[10] N. Petrochilos and P. Comon, "A zero-cumulant random variable and its applications," Signal Processing Magazine, 2006. Accepted.

[11] A. van der Veen and A. Paulraj, "An analytical constant modulus algorithm," IEEE Tr. Signal Processing, vol. 44, pp. 1136-1155, May 1996.

[12] A. van der Veen and J. Tol, "Separation of zero/constant modulus signals," in Proc. IEEE ICASSP, (Munich (FRG)), pp. 3445-3448, Apr. 1997.

[13] N. Petrochilos and A. van der Veen, "Algorithms to separate overlapping secondary surveillance radar replies," in Proc. IEEE ICASSP, pp. II.4953, May 2004.

[14] N. Petrochilos, G. Galati, L. Mené, and E. Piracci, "Separation of multiple secondary surveillance radar sources in a real environment by a novel projection algorithm," in Proc. ISSPIT 2005, Dec. 2005.

[15] D. Lawley, "Test of significance for the latent roots of covariance and correlation matrices," Biometrika, vol. 43, pp. 128-136, 1954.

[16] M. Wax and T. Kailath, "Detection of signals by information theoretic criteria," IEEE Trans. Acoust., Speech, Signal Processing, vol. 33, pp. 387-392, Apr. 1985.

[17] A. Liavas and P. Regalia, "On the behavior of information theoretic criteria for model order selection," IEEE Tr. Signal Proc., vol. 49, pp. 1689-1695, Aug. 2001

[18] P. Stoica and Y. Selen, "Model-order selection: A review of information criterion rules," IEEE Signal Processing Mag., vol. 21, no. 4, pp. 36-47, 2004.

[19] A. van der Veen, "Asymptotic properties of the algebraic constant modulus algorithm," IEEE Tr. Signal Processing, vol. 49, pp. 17961807, Aug. 2001.

[20] G. Golub and C. Van Loan, Matrix Computations. The Johns Hopkins University Press, 1989.

[21] J. Cardoso and A. Souloumiac, "Blind beamforming for non-Gaussian signals," IEE Proceedings-F, vol. 140, pp. 362-370, Dec. 1993.

[22] J.-F. Cardoso and A. Souloumiac, "Jacobi angles for simultaneous diagonalization," SIAM J. Matrix Anal. Appl., vol. 17, no. 1, pp. 161164, 1996. 
[23] L. D. Lathauwer, Signal Processing based on Multilinear Algebra. $\mathrm{PhD}$ thesis, K.U. Leuven, Leuven, Belgium, Sept. 1997.

[24] K. Abed-Meraim and Y. Hua, "A least-squares approach to joint Schur decomposition," in Proc. IEEE ICASSP, pp. 2541-2544, 1998.

[25] N. Sidiropoulos, G. Giannakis, and R. Bro, "Parallel factor analysis in sensor array processing," IEEE Trans. Signal Processing, vol. 48, pp. 2377-2388, Aug. 2000.

[26] A. Yeredor, "Non-orthogonal joint diagonalization in the least-squares sense with application in blind source separation," IEEE Tr. Signal Processing, vol. 50, July 2002.

[27] A. van der Veen, "Joint diagonalization via subspace fitting techniques," in Proc. IEEE ICASSP, (Salt Lake City (UT)), IEEE, May 2001.

[28] T. Beelen and P. V. Dooren, "An improved algorithm for the computation of Kronecker's canonical form of a singular pencil," Lin. Alg. Appl., vol. 105, pp. 9-65, 1988.

[29] A. van der Veen and A. Paulraj, "An analytical constant modulus algorithm,” IEEE Trans. Signal Processing, vol. 44, pp. 1136-1155, May 1996.

[30] A. Leshem, N. Petrochilos, and A. van der Veen, "Finite sample identifiability of multiple constant modulus sources," IEEE Tr. Information Theory, vol. 49, pp. 2314-2319, Sept. 2003.

[31] N. Petrochilos and P. Comon, "ML estimation of SSR signals, identifiability, and Cramer-Rao bounds," in Proc. EUSIPCO, (Tampere, Finland), Sept. 2000.

[32] A. Lemma, A. van der Veen, and E. Deprettre, "Analysis of joint angle-frequency estimation using ESPRIT," IEEE Tr. Signal Processing, vol. 51, pp. 1264-1283, May 2003.

[33] S. Kay, Fundamentals of statistical signal processing: estimation theory. Prentice hall, 1993.

[34] Z. Koldovsky and P. Tichavsky, "Efficient variant of algorithm fastica for independent component analysis attaining the Cramer-Rao lower bound," in IEEE/SP 13th workshop on Statistical Signal Processing, pp. 10901095, July 2005.

[35] R. Roy and T. Kailath, "ESPRIT_estimation of signal parameters via rotational invariance techniques," IEEE Tr. Acoustics, Speech, Signal Proc., vol. 37, pp. 984-995, July 1989.

[36] A. Belouchrani, K. A. Meraim, J. Cardoso, and E. Moulines, "A blind source separation technique based on second order statistics," IEEE Trans. Signal Processing, vol. 45, pp. 434-444, feb 1997.

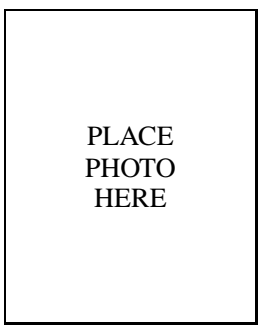

Nicolas Petrochilos was born in France in 1970 $\mathrm{He}$ got his bachelor in 1994 at "Ecole Normale Supérieure de Lyon". He obtained a Master of Science in Signal and Image Processing of E.N.S.E.A in Cergy-Pontoise in 1996, co-promoted by the E.N.S. Lyon, with the distinctions of the jury and was ranked first in his class. In 1997, he obtained a Master of Pedagogy in Physics. In December 2002, he obtained a Ph.D. degree from Technical University Delft, Netherland, and University of Nice, France. Throughout 2003 till 2004, he held a postdoc position at the University of Saint-Etienne (France). In fall 2004, he was a visiting professor at University of Tor Vergata. At present, he is an assistantprofessor at the University of Reims, on sabbatical leave at the University of Hawai'i at Mānoa, HI. His research interests are algebraic methods for array signal processing.

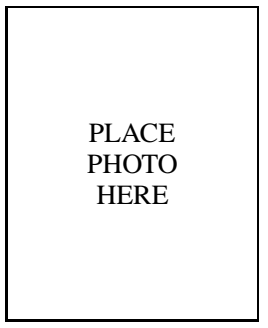

Alle-Jan van der Veen (M'95, SM'03, F'05) was born in The Netherlands in 1966 . He received the $\mathrm{Ph} . \mathrm{D}$. degree (cum laude) from TU Delft in 1993. Throughout 1994, he was a postdoctoral scholar at Stanford University. At present, he is a Full Professor in Signal Processing at TU Delft. He is the recipient of a 1994 and a 1997 IEEE Signal Processing Society (SPS) Young Author paper award, and was an Associate Editor for IEEE Tr. Signal Processing (1998-2001), chairman of IEEE SPS Signal Processing for Communications Technical Committee (2002-2004), and Editor-in-Chief of IEEE Signal Processing Letters (2002-2005). He currently is Editor-in-Chief of IEEE Transactions on Signal Processing, and member-at-large of the Board of Governors of IEEE SPS. His research interests are in the general area of system theory applied to signal processing, and in particular algebraic methods for array signal processing, with applications to wireless communications and radio astronomy. 